\title{
Uniform Error Estimates of Galerkin Methods for Monotone Abel-Volterra Integral Equations on the Half-Line*
}

\author{
By P. P. B. Eggermont \\ Dedicated to Prof. Dr. Gerhard Veltkamp on the occasion of his 65 th birthday
}

\begin{abstract}
We consider Galerkin methods for monotone Abel-Volterra integral equations of the second kind on the half-line. The $L^{2}$ theory follows from Kolodner's theory of monotone Hammerstein, equations. We derive the $L^{\infty}$ theory from the $L^{2}$ theory by relating the $L^{2}$ - and $L^{\infty}$-spectra of operators of the form $x \rightarrow b *(a x)$ to one another. Here * denotes convolution, and $b \in L^{1}$ and $a \in L^{\infty}$. As an extra condition we need $b(t)=O\left(t^{-\alpha-1}\right)$, with $\alpha>0$. We also prove the discrete analogue. In particular, we verify that the Galerkin matrix satisfies the "discrete" conditions.
\end{abstract}

1. Introduction. In this paper we study Galerkin methods for the approximate solution of monotone Abel-Volterra integral equations of the form

$$
x(t)+\lambda \int_{0}^{t}(t-s)^{\alpha-1} g(s, x(s)) d s=y(t), \quad t>0,
$$

where $0<\alpha<1$ and $g$ is a "nice" function of its arguments, with $g(s, 0)=0$ for all $s \in \mathbb{R}^{+}$, and

$$
\begin{aligned}
& \operatorname{Re} \overline{\left(x_{1}-x_{2}\right)}\left(g\left(s, x_{1}\right)-g\left(s, x_{2}\right)\right) \geq \delta\left|x_{1}-x_{2}\right|^{2} \\
& \quad \text { for all }\left(s, x_{i}\right) \in \mathbf{R}^{+} \times \mathbb{C}, \\
& \left|g\left(s, x_{1}\right)-g\left(s, x_{2}\right)\right| \leq D\left|x_{1}-x_{2}\right| \quad
\end{aligned}
$$

for some positive constants $D, \delta$, and where $|\arg \lambda| \leq(1-\alpha) \pi / 2$. We assume that $g(s, \sigma+i \tau)$ is at least continuously differentiable with respect to the real variables $\sigma$ and $\tau$. In particular, we assume that (1.17) holds, see below. Our insistence on solving Eq. (1.1) on all of $(0, \infty)$ is really just shorthand for writing that we want to solve (1.1) on some finite interval $[0, T]$ without any size restrictions on $\lambda$ or $T$ (and the mesh width in the Galerkin methods).

The Galerkin methods we consider here use subspaces of $C^{(-1)}$ piecewise polynomial functions on uniform grids. Accurate $L^{2}$ error estimates for the error in the Galerkin approximations are easily derived from the monotonicity of the Abel transform, which is preserved by the Galerkin approximation scheme, and the Hammerstein-Kolodner theory [15]. However, we are particularly interested in $L^{\infty}$

Received July 2, 1987; revised March 31, 1988.

1980 Mathematics Subject Classification (1985 Revision). Primary 65R20; Secondary 45D05, $47 \mathrm{~A} 10$.

* Research supported in part by the United States Army under contracts DAAG 29-83-K-0109 and DAAG 29-85-G-0009. Part of this research was performed while the author was visiting the Centre for Mathematics and Computer Science, Amsterdam, The Netherlands. Their hospitality is gratefully acknowledged. 
(uniform) error estimates, which follow once the solvability of (1.1) and the Galerkin equations are properly understood. We are able to do this by virtue of Hadamard's theorem, see e.g., Berger [3, (5.1.5)], which allows us to linearize Eq. (1.1), and essentially by showing that the spectrum of the operator $\mathbf{B}: L^{p}(0, \infty) \rightarrow L^{p}(0, \infty)$ defined by

$$
\mathbf{B} x(t)=\int_{0}^{t} b(t-s) a(s) x(s) d s, \quad t>0,
$$

where $a \in L^{\infty}(0, \infty), b \in L^{1}(0, \infty)$, and $b(t)=O\left(t^{-\alpha-1}\right)$ for $t \rightarrow \infty$, is virtually the same for all $L^{p}$ spaces. We prove that the $L^{\infty}$-spectrum is contained in the $L^{2}$-spectrum. This last result would appear to be of independent interest. The error estimates, both in $L^{2}$ and $L^{\infty}$, presuppose that the solution $x(t)$ is smooth everywhere. Since typically the solution is not smooth near $t=0$, even (especially) when $y$ is smooth, the full power of the Galerkin methods shows only when $\lambda$ is large (since $x$ is effectively nonsmooth only on an interval $[0, a]$ where $a$ is of the order $\lambda^{-1 / \alpha}$, or when $\lambda$ is small and singularity subtraction is used, as in [11]. The alternative is of course to use variable mesh widths as proposed by Brunner [4].

Equations of type (1) arise mostly in connection with parabolic differential equations, see Cannon [7] for references, but also in wave propagation phenomena when parabolic approximations are appropriate, see, e.g., Hufford [13], Mei and Tuck [20]. For numerical illustration we use the equation from Paveri-Fontana and Rigacci [22],

$$
x(t)+\lambda \int_{0}^{t}(t-s)^{-1 / 2} \frac{x(s)}{1-\theta x(s)} d s=1, \quad t>0,
$$

where $\lambda>0$ and $\theta \in[0,1)$. Here, $\lambda$ acts as a time scale parameter, and $\theta$ acts as a stiffness parameter.

The material in this paper has been discussed by Nevanlinna [21], in the $L^{2}$ setting, for the trapezoidal product integration method. Related work is done in Lubich [18]. Work by Kershaw [14] is only concerned with a finite interval (without the first part of conditions (1.2)). For a thorough discussion of RungeKutta methods for monotone (Abel) Volterra equations, see Brunner and van der Houwen [5]. Our approach here is based on work by Kolodner [15] on Hammerstein equations in Hilbert space setting. For the trapezoidal method for Eq. (1.1), this theory can be applied with minor modifications to obtain $L^{\infty}$ error estimates, see [9], but the argument cannot be extended to treat the general Galerkin scheme. It appears our approach here would also provide for uniform error estimates for Lubich's "fractional" methods [17] applied to nonlinear equations of the above type.

We give a brief outline of the remainder of this paper. In Section 2 we describe the Galerkin equations. The $L^{2}$ theory of the original equation and the Galerkin equations follows in Section 3. In Section 4 we prove the statement about the spectrum of the operator $\mathbf{B}$ defined in (1.3). In effect, we prove that the statement holds uniformly in $a \in L^{\infty}(0, \infty),\|a\|_{L^{\infty}(0, \infty)} \leq M$. In Section 5 we apply this to obtain the $L^{\infty}$ theory from the $L^{2}$ theory. The error estimates then follow in Section 6, with numerical illustrations in Section 7. The Appendix is devoted to proving that the discrete Galerkin equations satisfy the conditions of the discrete "spectrum theorem". 
We conclude this section with some notations. The set of complex numbers is denoted by $\mathbb{C}$. We set $\mathbf{R}^{+}=[0, \infty)$, and let $L^{p}\left(\mathbf{R}^{+}\right), 1 \leq p \leq \infty$, denote the Banach space of (equivalence classes of) measurable functions on $\mathbf{R}^{+}$, the $p$ th powers of which are Lebesgue integrable with norm

$$
\|f\|_{L^{p}\left(\mathbf{R}^{+}\right)}=\left\{\int_{0}^{\infty}|f(x)|^{p} d x\right\}^{1 / p} .
$$

For $p=\infty$ this should be interpreted as

$$
\|f\|_{L^{\infty}\left(\mathbf{R}^{+}\right)}=\operatorname{ess} \sup \left\{|f(x)|: x \in \mathbf{R}^{+}\right\} .
$$

If $T$ is a bounded linear operator on $L^{p}\left(\mathbf{R}^{+}\right)$, we define its norm as

$$
\|T\|_{L^{p}\left(\mathbf{R}^{+}\right)}=\sup \left\{\|T x\|_{L^{p}\left(\mathbf{R}^{+}\right)}:\|x\|_{L^{p}\left(\mathbf{R}^{+}\right)}=1\right\} \text {. }
$$

We let $l^{p}$ denote the Banach space of all infinite sequences of vectors in $\mathbb{C}^{P+1}$, the $p$ th power of which are summable, with norm

$$
\|x\|_{l p}=\left\{\sum_{i=0}^{\infty} \sum_{q=0}^{P}\left|x_{i q}\right|^{p}\right\}^{1 / p}, \quad 1 \leq p<\infty
$$

and for $p=\infty$

$$
\|x\|_{l \infty}=\sup \left\{\left|x_{i q}\right|: i \geq 0,0 \leq q \leq P\right\} .
$$

Equivalent norms, used interchangeably, are

$$
\begin{aligned}
\|x\|_{l p} & =\left\{\sum_{i=0}^{\infty}\left\|x_{i}\right\|^{p}\right\}^{1 / p}, \quad 1 \leq p<\infty \\
\|x\|_{l \infty} & =\sup \left\{\left\|x_{i}\right\|: i \geq 0\right\}
\end{aligned}
$$

where \|\| is any vector norm on $C^{P+1}$. When $T$ is a bounded linear operator on $l^{p}$, its norm $\|T\|_{l p}$ is defined analogously to (1.7).

In $L^{2}\left(\mathbf{R}^{+}\right), l^{2}$ and $\mathbb{C}^{P+1}$ we define inner products by

$$
\begin{aligned}
& (x, y)_{L^{2}\left(\mathbf{R}^{+}\right)}=\int_{0}^{\infty} \overline{x(t)} y(t) d t \quad \text { for } x, y \in L^{2}\left(\mathbf{R}^{+}\right) \\
& (x, y)_{l^{2}}=\sum_{i=0}^{\infty}\left(x_{i}, y_{i}\right)_{\mathbf{C}^{P+1}} \quad \text { for } x, y \in l^{2} \\
& (x, y)_{\mathbf{C}^{P+1}}=\sum_{q=0}^{P} \overline{x_{q}} y_{q} \quad \text { for } x, y \in \mathbb{C}^{P+1}
\end{aligned}
$$

Here the overhead bar denotes complex conjugation.

We let $C^{p}\left(\mathbf{R}^{+}\right)$denote the Banach space of all bounded functions on $\mathbf{R}^{+}$with bounded derivatives up to order $p$ and norm

$$
\|x\|_{C^{p}\left(\mathbf{R}^{+}\right)}=\sum_{i=0}^{p}\left\|\frac{\partial^{i} x}{\partial t^{i}}\right\|_{L^{\infty}\left(\mathbf{R}^{+}\right)} .
$$

Similarly, $C^{p}\left(\mathbf{R}^{+} \times \mathbb{C}\right)$ denotes the space of all functions bounded on $\mathbf{R}^{+} \times \mathbb{C}$, with continuous and bounded (real) partial derivatives up to order $p$ and norm

$$
\|x\|_{C^{p}\left(\mathbf{R}^{+} \times \mathbf{C}\right)}=\sum \sup \left\{\left|\frac{\partial^{k+l+m} x(r, t+i s)}{\partial r^{k} \partial t^{l} \partial s^{m}}\right|:(r, t+i s) \in \mathbf{R}^{+} \times \mathbb{C}\right\},
$$

where the summation is over all nonnegative integers $k, l, m$ with $k+l+m \leq p$. 
Finally, we write Eq. (1.1) as $x+\lambda \mathbf{A G}(x)=y$ where $\mathbf{A}$ is the Abel transform

$$
\mathbf{A} x(t)=\int_{0}^{t}(t-s)^{\alpha-1} x(s) d s, \quad t>0
$$

and

$$
\mathrm{G} x(t)=g(t, x(t)), \quad t>0 .
$$

Later on we have occasion to consider $g(t, \cdot)$ as a map from $\mathbb{C}$ into $\mathbb{C}$, for each $t$. We work out what the derivative map is. We write $\gamma(t, \sigma, \tau)=g(t, \sigma+i \tau)$ and $\gamma=\gamma_{1}+i \gamma_{2}$ where $\gamma_{1}$ and $\gamma_{2}$ are real-valued, and define the linear transformation $g^{\prime}(t, x)$ of $\mathbb{C}$ (considered as a real two-dimensional vector space) by

$$
g^{\prime}(t, x) h=h_{1} \frac{\partial \gamma_{1}}{\partial \sigma}+h_{2} \frac{\partial \gamma_{1}}{\partial \tau}+i\left(h_{1} \frac{\partial \gamma_{2}}{\partial \sigma}+h_{2} \frac{\partial \gamma_{2}}{\partial \tau}\right)
$$

where $h=h_{1}+i h_{2}$ with $h_{1}$ and $h_{2}$ real, and $x=\sigma+i \tau$. Here the partial derivatives are evaluated at $(t, x)$. We assume that uniformly in $t$

$$
\left|g\left(t, z_{1}\right)-g\left(t, z_{2}\right)-g^{\prime}\left(t, z_{2}\right)\left(z_{1}-z_{2}\right)\right|=o\left(\left|z_{1}-z_{2}\right|\right),
$$

and so $g^{\prime}(t, x)$ is the Fréchet derivative of $g(t, x)$. As an application we have that

$$
\operatorname{Re} \overline{\left(z_{1}-z_{2}\right)}\left(g\left(t, z_{1}\right)-g\left(t, z_{2}\right)\right)=\operatorname{Re} \overline{\left(z_{1}-z_{2}\right)} g^{\prime}\left(t, z_{3}\right)\left(z_{1}-z_{2}\right),
$$

where $z_{3}$ is some point on the line segment $\left[z_{1}, z_{2}\right]=\left\{t z_{1}+(1-t) z_{2}: 0 \leq t \leq 1\right\}$, and

$$
\left|g\left(t, z_{1}\right)-g\left(t, z_{2}\right)\right| \leq \sup \left|g^{\prime}(t, z)\right|\left|z_{1}-z_{2}\right|
$$

with the supremum over $z \in\left[z_{1}, z_{2}\right]$, see, e.g., Berger [3, (2.1.19)]. Here,

$$
\left|g^{\prime}(t, z)\right|=\sup \left\{\left|g^{\prime}(t, z) \xi\right|: \xi \in \mathbb{C},|\xi|=1\right\} .
$$

It follows from (1.2) and (1.17), (1.19) that for all $\xi \in \mathbb{C}, \xi \neq 0$, we have $\left(g^{\prime}(t, x) \xi\right) / \xi \in \Sigma$ with

$$
\Sigma=\{\varsigma \in \mathbb{C}: \operatorname{Re} \varsigma \geq \delta,|\varsigma| \leq D\} .
$$

2. The Galerkin Methods. Let $P$ be a nonnegative integer, which is arbitrary but fixed. Let $h>0$ be the mesh width parameter, let $\sigma_{i}=[i h,(i+1) h)$ for $i \geq 0$ be the subintervals of the resulting partition of $\mathbf{R}^{+}$, and let $S_{P}(h)$ be the space of bounded, piecewise polynomial functions of degree $\leq P$ on this partition,

$$
S_{P}(h)=\left\{x \in L^{\infty}\left(\mathbf{R}^{+}\right):\left.x\right|_{\sigma_{i}} \in \mathbf{P}_{P}, \text { for all } i \geq 0\right\} .
$$

Here $\mathbf{P}_{P}$ is the set of all polynomials of degree $\leq P$. We choose the usual "basis" in $S_{P}(h)$ as follows. Let $0 \leq u_{0}<u_{1}<\cdots<u_{P} \leq 1$, and let $l_{q}(t)$ be the fundamental Lagrange interpolating polynomials

$$
l_{q}(t)=\prod_{\substack{r=0 \\ r \neq q}}^{P} \frac{t-u_{r}}{u_{q}-u_{r}}
$$

and set

$$
l_{i q}(t)= \begin{cases}l_{q}(t / h-i), & t \in \sigma_{i}, \\ 0, & \text { otherwise. }\end{cases}
$$


Then every $x \in S_{P}(h)$ may be written as

$$
x(t)=\sum_{i=0}^{\infty} \sum_{q=0}^{P} x\left(t_{i q}\right) l_{i q}(t)
$$

where $t_{i q}=\left(i+u_{q}\right) h$. If $u_{q}=0$ or 1 , then right resp. left limits must be taken for $x\left(t_{i q}\right)$. Note that the infinite series in (2.4) reduces to a finite sum for every $t$, so there are no convergence problems.

It is natural to define the sampling and interpolating operators $r_{h}$ and $p_{h}$ by

$$
\left\{\begin{array}{l}
r_{h} x=\left(x_{0}^{T}, x_{1}^{T}, x_{2}^{T}, \ldots\right)^{T} \\
x_{i}=\left(x\left(t_{i 0}\right), x\left(t_{i 1}\right), \ldots, x\left(t_{i P}\right)\right)^{T}
\end{array}\right.
$$

and

$$
p_{h} r_{h} x(t)=\sum_{i=0}^{\infty} \sum_{q=0}^{P} x\left(t_{i q}\right) l_{i q}(t)
$$

for every $x \in S_{P}(h) \cup C\left(\mathbf{R}^{+}\right)$.

The Galerkin methods under consideration are embodied by the following system of equations:

$$
\left\{\begin{array}{l}
\left(l_{i q}, x_{h}+\lambda \mathbf{A G}\left(x_{h}\right)\right)=\left(l_{i q}, y\right) \text { for all } i, q, \\
x_{h} \in S_{P}(h)
\end{array}\right.
$$

which we immediately replace by its fully discretized version

$$
\left\{\begin{array}{l}
\left(l_{i q}, x_{h}+\lambda \mathbf{A} p_{h} r_{h} \mathbf{G}\left(x_{h}\right)\right)=\left(l_{i q}, y\right) \text { for all } i, q, \\
x_{h} \in S_{P}(h) .
\end{array}\right.
$$

Here $(\cdot, \cdot)$ is the $L^{2}$ inner product on $\mathbf{R}^{+}$. In the sequel, when we refer to the Galerkin approximation scheme or the Galerkin approximant $x_{h}$, this pertains to system (2.8) and its solution $x_{h}$ (assuming it exists).

In the remainder of this paper we consider the following questions: Do the systems (2.8) have unique solutions $x_{h} \in L^{\infty}\left(\mathbf{R}^{+}\right)$whenever $y \in L^{\infty}\left(\mathbf{R}^{+}\right)$, and do they depend continuously on $y$ ? How well do the $x_{h}$ approximate the solution $x(t)$ of the system (1.1)? We obtain completely satisfactory answers to these questions.

For numerical and analytical purposes we elucidate the structure of the system (2.8). When $y$ is a smooth function, which we will assume from now on, we may approximate $(2.8)$ as

$$
L r_{h} x_{h}+\lambda h^{\alpha} A G\left(r_{h} x_{h}\right)=L r_{h} y
$$

where $G\left(r_{h} x_{h}\right)=r_{h} \mathbf{G}\left(x_{h}\right)$, and $L$ and $A$ are block matrices with blocks $L_{i j}, A_{i j} \in$ $\mathbf{R}^{(P+1) \times(P+1)}$, and $L_{i j q r}=h^{-1}\left(l_{i q}, l_{j r}\right)_{L^{2}\left(\mathbf{R}^{+}\right)}$

$$
A_{i j q r}=\int_{0}^{1} l_{q}(t) \int_{0}^{1}\left(i-j+u_{q}-s\right)_{+}^{\alpha-1} l_{r}(s) d s d t=\left(a_{i-j}\right)_{q r} .
$$

Here $(t)_{+}^{\alpha-1}=0$ for $t \leq 0$ and $=t^{\alpha-1}$ for $t>0$. Note that $L$ and $A$ are independent of $h$, that $L$ is block diagonal, and that $A$ is block semicirculant, i.e., $A_{i j}$ depends only on $i-j$ and vanishes for $j>i$. Consequently, the system (2.9) is a block triangular system of nonlinear equations and can be solved by back substitution, 
at each stage of which a $(P+1) \times(P+1)$ system of nonlinear equations needs to be solved.

As an added simplification, both analytically and computationally, we assume that the $u_{q}, q=0,1, \ldots, P$, are the Gauss points for the interval $[0,1]$, in which case $L$ is a diagonal matrix, with

$$
L_{i i q q}=w_{q}, \quad q=0,1, \ldots, P,
$$

where the $w_{q}$ are the Gaussian weights for the interval $(0,1)$.

Since $L^{-1}$ is symmetric and positive definite, whether $L$ is diagonal or not, it has a unique symmetric and positive definite square root, which we denote by $M$,

$$
M=L^{-1 / 2} \text {. }
$$

3. The $L^{2}$ Setting. In this section we collect some results on the solvability of Eq. (1.1) and the Galerkin equations in the $L^{2}$ setting. This will be the stepping stone to the $L^{\infty}$ theory.

The first point of concern is the fact that the Abel transform $\mathbf{A}$ is not bounded on $L^{2}\left(\mathbf{R}^{+}\right)$. However we have

LEMMA 3.1. For all $\lambda \in \mathbb{C}$ with $|\arg \lambda| \leq(1-\alpha) \pi / 2$, the operator $\lambda \mathbf{A}$ is maximally monotone, i.e.,

(i) $\operatorname{Re}(x, \lambda \mathbf{A} x)_{L^{2}\left(\mathbf{R}^{+}\right)} \geq 0$ for all $x \in D_{\mathbf{A}}$, the domain of $\mathbf{A}$;

(ii) Range $(\mathbf{I}+\lambda \mathbf{A})=L^{2}\left(\mathbf{R}^{+}\right)$;

and $(\mathbf{I}+\lambda \mathbf{A})^{-1}$ is a bounded operator on $L^{2}\left(\mathbf{R}^{+}\right)$, and

$$
\left\|(\mathbf{I}+\lambda \mathbf{A})^{-1}\right\|_{L^{2}\left(\mathbf{R}^{+}\right)} \leq 1, \quad\left\|\lambda(\mathbf{I}+\lambda \mathbf{A})^{-1} \mathbf{A}\right\|_{L^{2}\left(\mathbf{R}^{+}\right)} \leq 1 .
$$

Remark. Strictly speaking, $\left\|\lambda(\mathbf{I}+\lambda \mathbf{A})^{-1} \mathbf{A} x\right\|_{L^{2}\left(\mathbf{R}^{+}\right)} \leq\|x\|_{L^{2}\left(\mathbf{R}^{+}\right)}$only for $x \in$ $D_{\mathbf{A}}$. Since for such $x$ we have $\lambda(\mathbf{I}+\lambda \mathbf{A})^{-1} \mathbf{A} x=x-(\mathbf{I}+\lambda \mathbf{A})^{-1} x$, we can extend $\lambda(\mathbf{I}+\lambda \mathbf{A})^{-1} \mathbf{A}$ to all of $L^{2}\left(\mathbf{R}^{+}\right)$in this manner.

Proof of Lemma 3.1. We give an informal proof of (i), also showing that $D_{\mathbf{A}}$, the domain of $\mathbf{A}$, is dense in $L^{2}\left(\mathbf{R}^{+}\right)$. By Plancherel's relation, for $x \in D_{\mathbf{A}}$,

$$
(x, \mathbf{A} x)_{L^{2}\left(\mathbf{R}^{+}\right)}=(2 \pi)^{-1}\left(\hat{x},(\mathbf{A} x)^{-}\right)_{L^{2}(\mathbf{R})},
$$

where $\hat{x}$ is the Fourier transform of $x$,

$$
\hat{x}(\omega)=\int_{0}^{\infty} x(t) e^{-i \omega t} d t .
$$

Since $\mathbf{A} x$ is a convolution, we have $(\mathbf{A} x)^{-}(\omega)=\hat{a}(\omega) \hat{x}(\omega)$ with

$$
\hat{a}(\omega)=\int_{0}^{\infty} t^{\alpha-1} e^{-i \omega t} d t=\Gamma(\alpha)(i \omega)^{-\alpha},
$$

where the principal value of the power function is used. Consequently, we have $\arg \left((i \omega)^{-\alpha}\right)=-\operatorname{signum}(\omega) \pi \alpha / 2$, and from

$$
(x, \mathbf{A} x)_{L^{2}\left(\mathbf{R}^{+}\right)}=(2 \pi)^{-1} \Gamma(\alpha) \int_{-\infty}^{\infty}|\hat{x}(\omega)|^{2}(i \omega)^{-\alpha} d \omega
$$

it follows that for $x \in D_{\mathbf{A}}$,

$$
\left|\arg (x, \mathbf{A} x)_{L^{2}\left(\mathbf{R}^{+}\right)}\right| \leq \pi \alpha / 2
$$


and (i) follows. The same informal argument shows that $D_{\mathbf{A}}$ is dense in $L^{2}\left(\mathbf{R}^{+}\right)$: approximate $x \in L^{2}\left(\mathbf{R}^{+}\right)$by $x_{\varepsilon}, \varepsilon>0$, defined by $\hat{x}_{\varepsilon}(\omega)=\hat{x}(\omega)$ for $|\omega| \geq \varepsilon$ and $=0$ otherwise. Then $\hat{a}(\omega) \hat{x}_{\varepsilon}(\omega)=\left(\mathbf{A} x_{\varepsilon}\right)^{\uparrow}(\omega)$ is an element of $L^{2}\left(\mathbf{R}^{+}\right)$, and so $x_{\varepsilon} \in D_{\mathbf{A}}$. Also, again by Plancherel's relation,

$$
\left\|x-x_{\varepsilon}\right\|_{L^{2}\left(\mathbf{R}^{+}\right)}^{2}=\left\|\hat{x}-\hat{x}_{\varepsilon}\right\|_{L^{2}(\mathbf{R})}^{2}=(2 \pi)^{-1} \int_{-\varepsilon}^{\varepsilon}|\hat{x}(\omega)|^{2} d \omega \rightarrow 0 \quad \text { as } \varepsilon \rightarrow 0 .
$$

Thus, $D_{\mathbf{A}}$ is dense in $L^{2}\left(\mathbf{R}^{+}\right)$. The above arguments can easily be made rigorous, but we do not do so here.

From (i) it now follows that for all $x \in D_{\mathbf{A}}$,

$$
\operatorname{Re}(x, x+\lambda \mathbf{A} x)_{L^{2}\left(\mathbf{R}^{+}\right)} \geq\|x\|_{L^{2}\left(\mathbf{R}^{+}\right)}^{2},
$$

which implies that $(\mathbf{I}+\lambda \mathbf{A})^{-1}$ is bounded on $\operatorname{range}(\mathbf{I}+\lambda \mathbf{A})$, and that $\operatorname{range}(\mathbf{I}+\lambda \mathbf{A})$ is closed in $L^{2}\left(\mathbf{R}^{+}\right)$. Since $D_{\mathbf{A}}$ is dense in $L^{2}\left(\mathbf{R}^{+}\right)$, inequality (3.4) then shows that range $(\mathbf{I}+\lambda \mathbf{A})=L^{2}\left(\mathbf{R}^{+}\right)$. (Otherwise, an $x \in L^{2}\left(\mathbf{R}^{+}\right), x \neq 0$, would exist which is orthogonal to range $(\mathbf{I}+\lambda \mathbf{A})$, violating (3.4).) So now $(\mathbf{I}+\lambda \mathbf{A})^{-1}$ is bounded on $L^{2}\left(\mathbf{R}^{+}\right)$. The inequalities (3.1) are then easy consequences.

We now discuss the solution of Eq. (1.1), following Kolodner [15]. With the help of Lemma 3.1 we see that Eq. (1.1) is equivalent (see also the Remark following Corollary 3.3) to the equation

$$
x+\lambda \nu(\mathbf{I}+\lambda \nu \mathbf{A})^{-1} \mathbf{A} \mathbf{G}_{\nu}(x)=(\mathbf{I}+\lambda \nu \mathbf{A})^{-1} y,
$$

where $\nu>0$ is an arbitrary real constant, and

$$
\mathbf{G}_{\nu}(x)=(\mathbf{G}(x)-\nu x) / \nu .
$$

The constant $\nu$ can be chosen such that $\mathrm{G}_{\nu}$ is a strong contraction as follows. In view of (1.15)-(1.20) we write

$$
\left|\left[\mathbf{G}_{\nu}\left(x_{1}\right)\right](t)-\left[\mathbf{G}_{\nu}\left(x_{2}\right)\right](t)\right| \leq \sup \left|\left[\mathbf{G}_{\nu}^{\prime}(x)\right](t)\right|\left|x_{1}(t)-x_{2}(t)\right|, \quad \text { a.e. } t,
$$

where

$$
\left[\mathbf{G}_{\nu}^{\prime}(x)\right](t)=\nu^{-1}\left(g^{\prime}(t, x(t))-\nu I\right)
$$

in which $I$ is the identity map on $\mathbb{C}$. Now recall that for all $z, \xi \in \mathbb{C}$ with $\xi \neq 0$ we have $\left(g^{\prime}(t, x) \xi\right) / \xi \in \Sigma$. Since $\Sigma$ lies in the halfplane $\operatorname{Re} \varsigma \geq \delta$, we can find a circle in the halfplane $\operatorname{Re} \varsigma \geq \delta / 2$ with center on the real line such that $\Sigma$ lies inside this circle. This says that for some $\nu>0$ (large enough) we have

$$
|\varsigma-\nu| \leq \nu-\delta / 2 \text { for all } \varsigma \in \Sigma \text {. }
$$

Consequently, for all $z, \xi \in \mathbb{C}, \xi \neq 0$,

$$
\left|\left(g^{\prime}(t, x) \xi-\nu \xi\right) / \xi\right| \leq \nu-\delta / 2 \text {. }
$$

It follows that for almost every $t$, and every $x \in L^{2}\left(\mathbf{R}^{+}\right)$,

$$
\left|\left[\mathbf{G}_{\nu}^{\prime}(x)\right](t)\right|=\sup _{|\xi|=1}\left|\left[\mathbf{G}_{\nu}^{\prime}(x)\right](t) \xi\right| \leq 1-\delta / 2 \nu .
$$

We now see that the operator $\lambda \nu(\mathbf{I}+\lambda \nu \mathbf{A})^{-1} \mathbf{A G}_{\nu}$ is a strong contraction, with contraction constant $1-\delta / 2 \nu$. By the Banach contraction principle, the solution of (3.5) exists. Moreover, if $x^{i}$ is the solution corresponding to $y^{i}$, then the above shows that

$$
\left\|x^{1}-x^{2}\right\|_{L^{2}\left(\mathbf{R}^{+}\right)} \leq 2 \nu / \delta\left\|y^{1}-y^{2}\right\|_{L^{2}\left(\mathbf{R}^{+}\right)},
$$


so that the solution $x$ depends Lipschitz continuously on $y$. We have thus proven:

THEOREM 3.2. With $\lambda, \mathbf{G}$ as in (1.1), the equation

$$
x+\lambda \mathbf{A G}(x)=y
$$

has a unique solution $x \in L^{2}\left(\mathbf{R}^{+}\right)$for every $y \in L^{2}\left(\mathbf{R}^{+}\right)$, and the solution depends Lipschitz continuously on $y$.

We also have the

COROLlaRY 3.3. With $\lambda, \mathbf{G}$ as in (1.1) and $\mathbf{G}_{\nu}$ given by (3.6), and for $\nu>0$ large enough but fixed, and for all $z \in L^{2}\left(\mathbf{R}^{+}\right)$, there holds

$$
\left\|\left[\mathbf{I}+\lambda \nu(\mathbf{I}+\lambda \nu \mathbf{A})^{-1} \mathbf{A G}_{\nu}^{\prime}(z)\right]^{-1}\right\|_{L^{2}\left(\mathbf{R}^{+}\right)} \leq \gamma,
$$

where $\gamma$ is independent of $\lambda$ or $z$.

It should be remarked that the corollary still holds for all measurable functions $z$, e.g., for all $z \in L^{\infty}\left(\mathbf{R}^{+}\right)$.

Remark. It is clear that if $x$ is a solution of (1.1), then it also solves (3.5). That the converse also holds can be seen as follows. The fact that $\mathbf{G}(x)=\nu\left(x+\mathbf{G}_{\nu}(x)\right)$ and $\mathbf{G}_{\nu}(x)$ is a strong contraction implies that $\mathbf{G}$ is an isomorphism of $L^{2}\left(\mathbf{R}^{+}\right)$. It follows that if $x \in L^{2}\left(\mathbf{R}^{+}\right)$is a solution of (3.5) in the sense that

$$
x+\left[\mathbf{I}-(\mathbf{I}+\lambda \nu \mathbf{A})^{-1}\right] \mathbf{G}_{\nu}(x)=(\mathbf{I}+\lambda \nu \mathbf{A})^{-1} y
$$

then

$$
\mathbf{G}(x)=\nu(\mathbf{I}+\lambda \nu \mathbf{A})^{-1}\left(y+\mathbf{G}_{\nu}(x)\right) .
$$

Since $\mathbf{G}_{\nu}(x) \in L^{2}\left(\mathbf{R}^{+}\right)$, this implies that $\mathbf{G}(x) \in D_{\mathbf{A}}$, and then it follows that $x$ solves (1.1) as well. So Eqs. (1.1) and (3.5) are indeed equivalent.

The above goes through for the Galerkin equations in essentially the same manner as for the continuous equation (1.1). Again we must worry first about the nonboundedness of $A$ on $l^{2}$.

LEMMA 3.4. For all $\mu \in \mathbb{C}$ with $|\arg \mu| \leq(1-\alpha) \pi / 2$, the operator $\mu A$ is maximally monotone on $l^{2}$, i.e.,

(i) $\operatorname{Re}(x, \mu A x)_{l^{2}} \geq 0$ for all $x \in D_{A}$;

(ii) Range $(L+\mu A)=l^{2}$;

and $(L+\mu A)^{-1}$ is a bounded operator on $l^{2}$, and

$$
\left\|(I+\mu M A M)^{-1}\right\|_{l^{2}} \leq 1, \quad\left\|(I+\mu M A M)^{-1} M A M\right\|_{l^{2}} \leq 1,
$$

where $M=L^{-1 / 2}$, see (2.12).

Proof. Again the crucial point is to establish (i), as well as the fact that $D_{A}$ is dense in $l^{2}$.

Let $h>0$, and let $x \in D_{A}$; then

$$
h^{\alpha+1}(x, \mu A x)_{l^{2}}=\left(p_{h} x, \mu \mathbf{A} p_{h} x\right)_{L^{2}\left(\mathbf{R}^{+}\right)}
$$

so by Lemma 3.1(i), part (i) of Lemma 3.4 follows. To show that $D_{A}$ is dense in $l^{2}$, let $x \in l^{2}$ and define $x_{\varepsilon}, \varepsilon>0$, by the requirement that

$$
\hat{x}_{\varepsilon}\left(e^{i \phi}\right)= \begin{cases}0 & \text { for }-\varepsilon<\phi<\varepsilon \\ \hat{x}\left(e^{i \phi}\right) & \text { for } \varepsilon<|\phi|<\pi\end{cases}
$$


where

$$
\hat{x}\left(e^{i \phi}\right)=\sum_{n=0}^{\infty} x_{n} e^{i n \phi} .
$$

Then by Parseval's relation,

$$
\left\|A x_{\varepsilon}\right\|_{l^{2}}^{2}=(2 \pi)^{-1} \int_{-\pi}^{\pi}\left\|\hat{a}\left(e^{i \phi}\right) \hat{x}_{\varepsilon}\left(e^{i \phi}\right)\right\|^{2} d \phi
$$

where $\|\cdot\|$ is the Euclidean norm on $\mathbb{C}^{P+1}$, and $\hat{a}\left(e^{i \phi}\right)$ has coefficients $a_{n}$, see (2.10). It is easy to show that $\hat{a}\left(e^{i \phi}\right)$ is bounded on $\varepsilon<|\phi|<\pi$, thus $A x_{\varepsilon} \in l^{2}$. Also $\left\|x-x_{\varepsilon}\right\|_{l^{2}} \rightarrow 0$ for $\varepsilon \rightarrow 0$, so that $D_{A}$ is dense in $l^{2}$.

From (i) it follows that

$$
\operatorname{Re}(x, x+\mu M A M x)_{l^{2}} \geq\|x\|_{l^{2}}^{2}
$$

and so, as in the proof of Lemma 3.1, range $(I+\mu M A M)=l^{2}$, and the estimates (3.9) follow. But now $L+\mu A=M^{-1}(I+\mu M A M) M^{-1}$, and so $\operatorname{range}(L+\mu A)=l^{2}$. This is part (ii) of the lemma.

THEOREM 3.5. With $\lambda, \mathbf{G}$ as in (1.1), the equation

$$
L x+\lambda h^{\alpha} A G(x)=y
$$

has a unique solution $x \in l^{2}$ for every $y \in l^{2}$, and the solution $x$ depends Lipschitz continuously on $y$ in the $l^{2}$ topology.

Proof. Let $\mu=\lambda h^{\alpha}$. The equation

$$
L x+\mu A G(x)=y
$$

is equivalent to

$$
z+\mu B H(z)=M y
$$

where $B=M A M$ and $H(z)=M^{-1} G(M z)$. Then, using Lemma 3.4, Eq. (3.13) is equivalent to

$$
z+\mu \nu(I+\mu \nu B)^{-1} B H_{\nu}(z)=(I+\mu \nu B)^{-1} y
$$

for $\nu>0$, where

$$
H_{\nu}(z)=\left(M^{-1} G(M z)-\nu z\right) / \nu .
$$

Note that the Fréchet derivative of $H(z)$ equals $H^{\prime}(z)=M^{-1} G^{\prime}(M z) M$. Since for $x, y \in l^{2}$

$$
\left[G^{\prime}(x) y\right]_{i q}=g^{\prime}\left(t_{i q}, x_{i q}\right) y_{i q} \text { for all } i, q
$$

and since $M$ is a diagonal matrix, we thus have that $H^{\prime}(z)=G^{\prime}(M z)$. The proof now proceeds as in the proof of Theorem 3.2, and is omitted.

COROLlaRY 3.6. Let $\lambda, \mathbf{G}$ be as in (1.1) and let $H_{\nu}$ be given by (3.15) with $\nu>0$ large enough. Then there exists a constant $\gamma$ such that for all $h>0$ and for all $z \in l^{2}$,

$$
\left\|\left[I+B_{\mu \nu} H_{\nu}^{\prime}(z)\right]^{-1}\right\|_{l^{2}} \leq \gamma .
$$

Here, $\mu=\lambda h^{\alpha}, B_{\mu \nu}=\mu \nu[I+\mu \nu M A M]^{-1} M A M$, and $M=L^{-1 / 2}$, cf. (2.12). 
The next step in the program is to repeat the above analysis for the $L^{\infty}$ setting. This cannot be done in an analogous manner, since the crucial inequalities (3.1) and (3.9) do not hold for $L^{\infty}\left(\mathbf{R}^{+}\right)$and $l^{\infty}$. In particular, the inequality

$$
\left\|\lambda(\mathbf{I}+\lambda \mathbf{A})^{-1} \mathbf{A}\right\|_{L^{\infty}\left(\mathbf{R}^{+}\right)} \leq 1
$$

fails for nonreal $\lambda$, even with $|\arg \lambda| \leq(1-\alpha) \pi / 2$. One redeeming feature is that the operator $\mathbf{B}_{\lambda}$ defined by

$$
\mathbf{B}_{\lambda}=\lambda(\mathbf{I}+\lambda \mathbf{A})^{-1} \mathbf{A}
$$

is a convolution operator with $L^{1}$ kernel, Kershaw [14],

$$
\mathbf{B}_{\lambda} x(t)=\int_{0}^{t} b_{\lambda}(t-s) x(s) d s, \quad t>0
$$

with

$$
b_{\lambda}(t)=-\frac{d}{d t}\left\{E_{\alpha}\left(-\lambda \Gamma(\alpha) t^{\alpha}\right)\right\}
$$

where $E_{\alpha}(z)$ is the Mittag-Leffler function, Erdélyi [12, Chapter 18]. Then we may write

$$
b_{\lambda}(t)=\mu B(\mu t)
$$

with $\mu=\lambda^{1 / \alpha}$, and

$$
B(t)=-\frac{d}{d t}\left\{E_{\alpha}\left(-\Gamma(\alpha) t^{\alpha}\right)\right\}=O\left(t^{-1-\alpha}\right), \quad t \rightarrow \infty
$$

It should be noted that the fact that $b_{\lambda} \in L^{1}\left(\mathbf{R}^{+}\right)$is actually closely related to the monotonicity of $\mathbf{A}$, see, e.g., Londen [16].

In the next section we investigate the spectra of the operators $B_{\lambda} \mathbf{G}^{\prime}(z)$ in the $L^{\infty}$ space, and then apply these results to obtain information about Eq. (1.1) in the $L^{\infty}$ setting.

4. On Convolution-Like Operators. Let $b \in L^{1}(\mathbf{R})$ and $e \in C(\mathbf{R})$ with $e(0)=0$. We consider the class $\mathbf{F}(b, e)$ of measurable functions $k$ on $\mathbf{R}^{2}$ for which there exists a positive constant $\mu$ such that

$$
|k(t, s)| \leq \mu b(\mu(t-s)) \text { a.e. } t, s \in \mathbb{R},
$$

and

$$
\sup _{t} \int_{-\infty}^{\infty}|k(t+h, s)-k(t, s)| d s \leq e(\mu h) .
$$

Note that if $k(t, s)=b(t-s) a(s)$ for $a \in L^{\infty}(\mathbf{R}),\|a\|_{L^{\infty}(\mathbf{R})} \leq 1$, then (4.1), (4.2) are satisfied for a suitable function $e$. With minor reinterpretations, this also covers the case where $k$ takes on values in the set of all linear transformations of $\mathbb{C}$ (considered as a two-dimensional vector space over the reals). We will not further elaborate this point.

Let $\Omega$ denote either $\mathbf{R}$ or $\mathbf{R}^{+}$. Then $k$ generates an integral operator $\mathbf{K}$ defined by

$$
\mathbf{K} x(t)=\int_{\Omega} k(t, s) x(s) d s, \quad t \in \Omega
$$


which by virtue of Young's inequality, Stein and Weiss [23], maps any $L^{p}(\Omega)$ into itself for $1 \leq p \leq \infty$, and for all $x \in L^{p}(\Omega)$ we have

$$
\|\mathbf{K} x\|_{L^{p}(\Omega)} \leq\|b\|_{L^{1}(\mathbf{R})}\|x\|_{L^{p}(\Omega)} .
$$

For $\Omega=\mathbb{R}^{+}$the operator $\mathbf{K}$ is either a Volterra operator or a Wiener-Hopf type operator, depending on whether $k(t, s)=0$ for all $s>t$ or not.

The class of operators $\mathbf{K}$ given by (4.3) for which (4.1), (4.2) holds for some $\mu>0$ is also denoted by $\mathbf{F}(b, e)$. For such operators we may define the spectrum with respect to $L^{p}(\mathbb{R})$ in the usual way by

$$
\sigma_{p}(\mathbf{K})=\left\{\lambda \in \mathbb{C}: \lambda \mathbf{I}-\mathbf{K} \text { has no bounded inverse on } L^{p}(\mathbf{R})\right\},
$$

and the resolvent set by $\rho_{p}(\mathbf{K})=\mathbb{C} \backslash \sigma_{p}(\mathbf{K})$. We are interested in obtaining $L^{\infty}$ existence theorems from the $L^{2}$ theory, so we would like to show that $\sigma_{2}(\mathbf{K})=$ $\sigma_{\infty}(\mathbf{K})$. But the prospective application of Hadamard's theorem to obtain the solvability of nonlinear equations forces us to consider families of such operators, so we need a slightly stronger result. To prove the following theorem we need the additional assumption

$$
b(t)=O\left(|t|^{-\alpha-1}\right), \quad t \rightarrow \pm \infty
$$

for some $\alpha>0$. We conjecture that the theorem is true without this condition, i.e., that "just" $b \in L^{1}(\mathbf{R})$ is sufficient.

THEOREM 4.1. Let $\mathbf{E} \subset \mathbf{F}(b, e)$ and let $\lambda \in \mathbb{C}$. If $\lambda \in \rho_{2}(\mathbf{K})$ for all $\mathbf{K} \in \mathbf{E}$ and

$$
\sup \left\|(\lambda \mathbf{I}-\mathbf{K})^{-1}\right\|_{L^{2}(\Omega)}<\infty
$$

then $\lambda \in \rho_{\infty}(\mathbf{K})$ for all $\mathbf{K} \in \mathbf{E}$ and

$$
\sup \left\|(\lambda \mathbf{I}-\mathbf{K})^{-1}\right\|_{L^{\infty}(\Omega)}<\infty .
$$

The suprema in (4.7) and (4.8) are taken over all $\mathbf{K} \in \mathbf{E}$.

The proof of Theorem 4.1 is broken up into two parts. First we consider the boundedness aspects, and then the invertibility, even if this seems to be the wrong way around.

LEMMA 4.2. Let $\mathbf{E} \subset \mathbf{F}(b, e)$ and $\lambda \in \mathbb{C}$. If there exists a constant $c>0$ such that for all $\mathbf{K} \in \mathbf{E}$,

$$
\|(\lambda \mathbf{I}-\mathbf{K}) x\|_{L^{2}(\Omega)} \geq c\|x\|_{L^{2}(\Omega)}
$$

for all $x \in L^{2}(\Omega)$, then likewise

$$
\|(\lambda \mathbf{I}-\mathbf{K}) x\|_{L^{\infty}(\Omega)} \geq c_{1}\|x\|_{L^{\infty}(\Omega)}
$$

for some constant $c_{1}>0$ and for all $\mathbf{K} \in \mathbf{E}$ and $x \in L^{\infty}(\Omega)$.

LEMMA 4.3. Let $\mathbf{K} \in \mathbf{F}(b, e)$; then

$$
\rho_{2}(\mathbf{K}) \subset \rho_{\infty}(\mathbf{K})
$$

Proving the equality of the resolvent sets turns out to be more involved. Fortunately, we do not need equality. We are now ready for the proof. 
Proof of Theorem 4.1. Evidently, (4.7) implies (4.9), and so by Lemma 4.2 we obtain (4.10). From Lemma 4.3 we obtain that $\lambda \in \rho_{\infty}(\mathbf{K})$ for all $\mathbf{K} \in$ $\mathbf{E}$, and thus $\lambda \mathbf{I}-\mathbf{K}$ is invertible on $L^{\infty}(\Omega)$. Combined with (4.10), this gives $\left\|(\lambda \mathbf{I}-\mathbf{K})^{-1}\right\|_{L^{\infty}(\Omega)} \leq c_{1}^{-1}$ for all $\mathbf{K} \in \mathbf{E}$, which is (4.8).

We now prove Lemma 4.2. We will actually use Lemma 4.2 in the proof of Lemma 4.3, so we really do consider the boundedness before the invertibility aspects.

Proof of Lemma 4.2. As a first step in the proof we note that the "free" parameter $\mu$ in (4.1), (4.2) acts in essence as a time scale parameter, but time scaling does not affect norms or spectra, i.e., if $\mathbf{K} \in \mathbf{F}(b, e)$, define $k_{\nu}$ by

$$
k_{\nu}(t, s)=\nu k(\nu t, \nu s), \quad t, s \in \mathbf{R},
$$

and define $\mathbf{K}_{\nu}$ as in (4.3). Then $\sigma_{p}(\mathbf{K})=\sigma_{p}\left(\mathbf{K}_{\nu}\right)$, and for $\lambda \notin \sigma_{p}(\mathbf{K})$,

$$
\left\|(\lambda \mathbf{I}-\mathbf{K})^{-1}\right\|_{L^{p}(\Omega)}=\left\|\left(\lambda \mathbf{I}-\mathbf{K}_{\nu}\right)^{-1}\right\|_{L^{p}(\Omega)},
$$

for all $1 \leq p \leq \infty$. It thus suffices to prove the theorem for subsets $\mathbf{E}$ of $\mathbf{F}(b, e)$ for which (4.1), (4.2) holds for $\mu=1$.

Secondly, we note that neither (4.9) nor (4.10) can hold for $\lambda=0$. We may thus assume that $\lambda \neq 0$.

Suppose that (4.10) does not hold. Then there exist sequences $\left\{\mathbf{K}_{n}\right\} \subset \mathbf{E}$ and $\left\{x_{n}\right\} \subset L^{\infty}(\Omega)$ with $\left\|x_{n}\right\|_{L^{\infty}(\Omega)}=1$ such that

$$
\left\|\lambda x_{n}-\mathbf{K}_{n} x_{n}\right\|_{L^{\infty}(\Omega)}=O\left(n^{-2}\right), \quad n \rightarrow \infty .
$$

We are now going to construct functions $a_{n} \in L^{2}(\Omega)$ such that $\left\|a_{n} x_{n}\right\|_{L^{2}(\Omega)}$ is bounded away from 0 and $\left\{\lambda a_{n} x_{n}-\mathbf{K}_{n} a_{n} x_{n}\right\}_{n}$ is a null sequence in $L^{2}(\Omega)$. It then follows that (4.9) does not hold, and the lemma is proved.

First observe that from (4.2) for all $n$ and for all $t \in \Omega$,

$$
\left|\mathbf{K}_{n} x_{n}(t+h)-\mathbf{K}_{n} x_{n}(t)\right| \leq e(h),
$$

so that each $\mathbf{K}_{n} x_{n}$ is uniformly continuous on $\Omega$, uniformly in $n$. Note that (4.11) implies that

$$
\left\|\mathbf{K}_{n} x_{n}\right\|_{L^{\infty}(\Omega)} \geq|\lambda|-O\left(n^{-2}\right),
$$

so that $\left\|\mathbf{K}_{n} x_{n}\right\|_{L^{\infty}(\Omega)}$ is bounded away from zero for large $n$. Now choose $t_{n} \in \Omega$ such that $\left|\mathbf{K}_{n} x_{n}\left(t_{n}\right)\right| \geq \frac{2}{3}|\lambda|$, and choose $d>0$ such that for all $t \in \Omega$ with $\left|t-t_{n}\right|<d$

$$
\left|\mathbf{K}_{n} x_{n}(t)\right| \geq \frac{1}{2}|\lambda|
$$

Note that $d$ can be chosen independent of $n$. Then again (4.11) implies that for $n$ large enough

$$
\left|x_{n}(t)\right| \geq \frac{1}{3}, \quad \text { a.e. } t \in \Omega,\left|t-t_{n}\right|<d .
$$

Now define $a_{n} \in L^{2}(\Omega)$ by

$$
a_{n}(t)=\left(1+\frac{\left|t-t_{n}\right|}{n}\right)^{-(1+\alpha) / 2}, \quad t \in \Omega .
$$

Observe that

$$
\left\|a_{n}\right\|_{L^{2}(\Omega)}^{2} \leq \int_{-\infty}^{\infty}\left(1+\frac{|t|}{n}\right)^{-1-\alpha} d t=\frac{2 n}{\alpha}
$$


so that from (4.11)

$$
\left\|\lambda a_{n} x_{n}-a_{n} \mathbf{K}_{n} x_{n}\right\|_{L^{2}(\Omega)}=O\left(n^{-3 / 2}\right),
$$

and also note that for all $n$ large enough

$$
\left\|a_{n} x_{n}\right\|_{L^{2}(\Omega)}^{2} \geq \int_{\left|t-t_{n}\right|<d}\left[\frac{1}{3} a_{n}(t)\right]^{2} d t \geq d^{*}
$$

where $d^{*}$ is some positive constant. From (4.14) and (4.15) we finally get that

$$
\frac{\left\|\lambda a_{n} x_{n}-a_{n} \mathbf{K}_{n} x_{n}\right\|_{L^{2}(\Omega)}}{\left\|a_{n} x_{n}\right\|_{L^{2}(\Omega)}}=O\left(n^{-3 / 2}\right) .
$$

Now SUPPOSE that for all $x \in L^{\infty}(\Omega)$,

$$
\left\|a_{n} \mathbf{K}_{n} x-\mathbf{K}_{n} a_{n} x\right\|_{L^{2}(\Omega)} \leq B_{n}\left\|a_{n} x\right\|_{L^{2}(\Omega)},
$$

where $B_{n} \rightarrow 0$ as $n \rightarrow \infty$; then (4.16) implies that

$$
\frac{\left\|\lambda a_{n} x_{n}-\mathbf{K}_{n} a_{n} x_{n}\right\|_{L^{2}(\Omega)}}{\left\|a_{n} x_{n}\right\|_{L^{2}(\Omega)}} \rightarrow 0 .
$$

It follows that (4.9) does not hold, and the lemma is proved. So it all comes down to proving (4.17).

Observe that $a_{n} \mathbf{K}_{n} x-\mathbf{K}_{n} a_{n} x=\left(a_{n} \mathbf{K}_{n} a_{n}^{-1}-\mathbf{K}_{n}\right) a_{n} x$, and that for $z \in L^{2}(\Omega)$,

$$
\left\|\left(a_{n} \mathbf{K}_{n} a_{n}^{-1}-\mathbf{K}_{n}\right) z\right\|_{L^{2}(\Omega)}^{2}=\int_{\Omega}\left|\int_{\Omega} k_{n}(t, s) l_{n}(t, s) z(s) d s\right|^{2} d t
$$

and so, using Cauchy-Schwarz, we may estimate this as

$$
M \int_{\Omega} \int_{\Omega} b(t-s)\left|l_{n}(t, s)\right|^{2-\sigma}|z(s)|^{2} d s d t
$$

with

$$
M=\sup \int_{\Omega} b(t-s)\left|l_{n}(t, s)\right|^{\sigma} d s,
$$

where $\sigma=\alpha /(\alpha+1)$ and

$$
l_{n}(t, s)=\frac{a_{n}(t)}{a_{n}(s)}-1
$$

Writing this out, we have

$$
\left|l_{n}(t, s)\right|=\left|\left(\frac{n+\left|s-t_{n}\right|}{n+\left|t-t_{n}\right|}\right)^{(1+\alpha) / 2}-1\right|
$$

and since $f(x)=x^{(1+\alpha) / 2}$ is Lipschitz continuous of order $(1+\alpha) / 2$, provided $(1+\alpha) / 2 \leq 1$, we see that

$$
\left|l_{n}(t, s)\right| \leq c\left|\frac{\left|s-t_{n}\right|-\left|t-t_{n}\right|}{n+\left|t-t_{n}\right|}\right|^{(1+\alpha) / 2} \leq c\left(\frac{|t-s|}{n}\right)^{(1+\alpha) / 2} .
$$

Now we get that

$$
M \leq c_{1} n^{-\alpha / 2} \int_{\mathbf{R}} b(t)|t|^{\alpha / 2} d t .
$$

By (4.6) the integral converges, thus $M=O\left(n^{-\alpha / 2}\right)$. 
Applying Fubini's Theorem to (4.19), we may interchange the order of integration and estimate to obtain

$$
\int_{\Omega}|z(s)|^{2}\left|a_{n}(s)\right|^{\sigma-2} \int_{\Omega} b(t-s)\left[\left|a_{n}(t)\right|^{2-\sigma}+\left|a_{n}(s)\right|^{2-\sigma}\right] d t d s .
$$

Consequently, if we can show that for some constant $c$

$$
\int_{\Omega} b(t-s)\left|a_{n}(t)\right|^{2-\sigma} d t \leq c\left|a_{n}(s)\right|^{2-\sigma}
$$

for all $s$, then the whole inner integral can be estimated like this, and the integral (4.20) can be estimated as $c\|z\|_{L^{2}(\Omega)}^{2}$ for some suitable constant $c$, and (4.17) follows. To prove (4.21), observe that we may take $\Omega=\mathbb{R}$ and that it suffices to prove it for $s>0$. Also, it suffices to prove (4.21) with $a_{n}(t)$ replaced by $A_{n}(t)=$ $(1+|t| / n)^{-(1+\alpha) / 2}$, i.e., we suppose here that all $t_{n}=0$, see (4.13). We split the integral in (4.21) according to $\mathbf{R}=(-\infty, s / 2) \cup[s / 2, \infty)$. Now, since $s>0$ and $A_{n}(t)$ is decreasing for $t>0$,

$$
\begin{aligned}
\int_{s / 2}^{\infty} b(t-s)\left|A_{n}(t)\right|^{2-\sigma} d t & \leq\left|A_{n}(s / 2)\right|^{2-\sigma} \int_{-\infty}^{\infty} b(t-s) d s \\
& \leq c\left|A_{n}(s)\right|^{2-\sigma}
\end{aligned}
$$

for a suitable constant $c$ independent of $s$ and $n$. Also, by (4.6), for $s \geq n$

$$
\begin{aligned}
\int_{-\infty}^{s / 2} b(t-s)\left|A_{n}(t)\right|^{2-\sigma} d t & \leq c s^{-\alpha-1} \int_{-\infty}^{\infty}\left|A_{n}(t)\right|^{2-\sigma} d t \\
& \leq c_{1} s^{-\alpha-1} n
\end{aligned}
$$

and one verifies that for all $n$ and all $s \geq n$,

$$
n s^{-\alpha-1}=n^{-\alpha}\left(\frac{s}{n}\right)^{-\alpha-1} \leq c n^{-\alpha}\left(1+\frac{s}{n}\right)^{-\alpha-1}
$$

and this is less than $c n^{-\alpha}\left|A_{n}(s)\right|^{2} \leq c n^{-\alpha}\left|A_{n}(s)\right|^{2-\sigma}$. For $0<s \leq n$ it suffices to observe that

$$
\int_{-\infty}^{\infty} b(t-s)\left|A_{n}(t)\right|^{2-\sigma} d t \leq\|b\|_{L^{1}(\mathbf{R})}
$$

Now (4.22)-(4.24) establish (4.21), and we are done.

Proof of Lemma 4.3. Let $\lambda \in \rho_{2}(\mathbf{K})$ and set $c=\left\|(\lambda \mathbf{I}-\mathbf{K})^{-1}\right\|_{L^{2}(\Omega)}$. Let $\mathbf{K}$ have kernel $k(t, s)$. For $n \in \mathbb{N}$ define the operator $\mathbf{K}_{n}$ as having the kernel $k_{n}(t, s)$ defined by

$$
k_{n}(t, s)= \begin{cases}0, & \text { if }|t-s|>n \text { or }|k(t, s)|>n, \\ k(t, s), & \text { otherwise. }\end{cases}
$$

Then for $n$ large enough, $\left\|\mathbf{K}-\mathbf{K}_{n}\right\|_{L^{p}(\Omega)} \leq 1 / 2 c$ for all $1 \leq p \leq \infty$, so that by the Banach contraction theorem, $\lambda \mathbf{I}-\mathbf{K}_{n}=(\lambda \mathbf{I}-\mathbf{K})\left[\mathbf{I}+(\lambda \mathbf{I}-\mathbf{K})^{-1}\left(\mathbf{K}-\mathbf{K}_{n}\right)\right]$ is invertible on $L^{2}(\Omega)$ and $\left\|\left(\lambda \mathbf{I}-\mathbf{K}_{n}\right)^{-1}\right\|_{L^{2}(\Omega)} \leq 2 c$. Let $\mathbf{L}_{n}$ be the integral operator on $L^{2}(\Omega)$ defined by

$$
\lambda^{-1} \mathbf{I}+\mathbf{L}_{n}=\left(\lambda \mathbf{I}-\mathbf{K}_{n}\right)^{-1} .
$$

Now consider the operator $\mathbf{K}_{n \varepsilon}$ defined by

$$
k_{n \varepsilon}(t, s)=e^{\varepsilon(t-s)} k_{n}(t, s) \quad \text { all } t, s .
$$


It follows that there exists an $\varepsilon_{n}$ such that for all $|\varepsilon| \leq \varepsilon_{n}$ we have

$$
\left\|\mathbf{K}_{n}-\mathbf{K}_{n \varepsilon}\right\|_{L^{p}(\Omega)} \leq 1 / 4 c
$$

for all $p, 1 \leq p \leq \infty$. For example, for $n$ large enough , $\varepsilon_{n}=n^{-2}$ would work. As before, $\lambda \mathbf{I}-\mathbf{K}_{n \varepsilon}$ is invertible on $L^{2}(\Omega)$, and $\left\|\left(\lambda \mathbf{I}-\mathbf{K}_{n \varepsilon}\right)^{-1}\right\|_{L^{2}(\Omega)} \leq 4 c$. If we define $\mathbf{L}_{n \varepsilon}$ by

$$
\lambda^{-1} \mathbf{I}+\mathbf{L}_{n \varepsilon}=\left(\lambda \mathbf{I}-\mathbf{K}_{n \varepsilon}\right)^{-1}
$$

then the relationship between its kernel $l_{n \varepsilon}(t, s)$ and the kernel $l_{n}(t, s)$ of $\mathbf{L}_{n}$ is, cf. (4.26),

$$
l_{n}(t, s)=e^{-\varepsilon(t-s)} l_{n \varepsilon}(t, s) \quad \text { all } t, s .
$$

The equation for $l_{n \varepsilon}(t, s)$ is, using Fubini's theorem,

$$
l_{n \varepsilon}(t, s)-\lambda^{-1} \int_{\Omega} k_{n \varepsilon}(t, r) l_{n \varepsilon}(r, s) d r=\lambda^{-2} k_{n \varepsilon}(t, s),
$$

from which it follows that for a.e. $s$, using (4.25-26) in the last inequality,

$$
\left\|l_{n \varepsilon}(\cdot, s)\right\|_{L^{2}(\Omega)} \leq 4 c \lambda^{-1}\left\|k_{n \varepsilon}(\cdot, s)\right\|_{L^{2}(\Omega)} \leq 4 c \lambda^{-1} n e^{|\varepsilon| n} \sqrt{2 n} .
$$

Taking it one step further, we get that

$$
\left|\int_{\Omega} k_{n \varepsilon}(t, r) l_{n \varepsilon}(r, s) d r\right| \leq\left\|k_{n \varepsilon}(t, \cdot)\right\|_{L^{2}(\Omega)}\left\|l_{n \varepsilon}(\cdot, s)\right\|_{L^{2}(\Omega)} \leq 8 c \lambda^{-1} n^{3} e^{2|\varepsilon| n},
$$

and thus from (4.29) we see that there exists a $c(n)$ such that

$$
\left|l_{n \varepsilon}(t, s)\right| \leq c(n) \quad \text { all } t, s .
$$

We then obtain from (4.28) that

$$
\left|l_{n}(t, s)\right| \leq c(n) e^{-\varepsilon(t-s)} .
$$

Now taking $\varepsilon=\varepsilon_{n}$ and $\varepsilon=-\varepsilon_{n}$, we get

$$
\left|l_{n}(t, s)\right| \leq c(n) e^{-\varepsilon_{n}|t-s|} .
$$

It follows that $\lambda^{-1} \mathbf{I}+\mathbf{L}_{n}$ is also a bounded operator on $L^{\infty}(\Omega)$, and then also that $\lambda^{-1} \mathbf{I}+\mathbf{L}_{n}=\left(\lambda \mathbf{I}-\mathbf{K}_{n}\right)^{-1}$ on $L^{\infty}(\Omega)$. This holds for all $n$ large enough.

Now for $\lambda \in \rho_{2}(\mathbf{K})$ we have $\|(\lambda \mathbf{I}-\mathbf{K}) x\|_{L^{2}(\Omega)} \geq c^{-1}\|x\|_{L^{2}(\Omega)}$, and thus also for all $n$ large enough

$$
\left\|\left(\lambda \mathbf{I}-\mathbf{K}_{n}\right) x\right\|_{L^{2}(\Omega)} \geq \frac{1}{2 c}\|x\|_{L^{2}(\Omega)}
$$

for all $x \in L^{2}(\Omega)$. Now if $\mathbf{K} \in \mathbf{F}(b, e)$, then also $\mathbf{K}_{n} \in \mathbf{F}(b, e)$ for all $n$, so that by Lemma 4.2,

$$
\left\|\left(\lambda \mathbf{I}-\mathbf{K}_{n}\right) x\right\|_{L^{\infty}(\Omega)} \geq c_{1}\|x\|_{L^{\infty}(\Omega)}
$$

for some constant $c_{1}$, and for all $x \in L^{\infty}(\Omega)$. Since we have shown above that $\lambda \mathbf{I}-\mathbf{K}_{n}$ is invertible on $L^{\infty}(\Omega)$ for all $n$ large enough, we thus conclude that

$$
\sup _{n}\left\|\left(\lambda \mathbf{I}-\mathbf{K}_{n}\right)^{-1}\right\|_{L^{\infty}(\Omega)} \leq c_{1}^{-1} \text {. }
$$

The Banach contraction theorem applied to

$$
\lambda \mathbf{I}-\mathbf{K}=\left(\lambda \mathbf{I}-\mathbf{K}_{n}\right)\left[\mathbf{I}-\left(\lambda \mathbf{I}-\mathbf{K}_{n}\right)^{-1}\left(\mathbf{K}-\mathbf{K}_{n}\right)\right]
$$

then finally gives us that $\lambda \mathbf{I}-\mathbf{K}$ is invertible on $L^{\infty}(\Omega)$. So $\lambda \in \rho_{\infty}(\mathbf{K})$. 
Finally, we state the discrete version of Theorem 4.1, which is in effect a discretized version. Let $b \in L^{1}(\mathbf{R})$ and $e \in C(\mathbf{R})$ with $e(0)=0$, as before. Let $k$ be a bounded function on $\mathbf{Z}^{2}$ for which for some $\mu>0$

$$
|k(m, n)| \leq \mu \int_{m-n}^{m-n+1} b(\mu t) d t
$$

and

$$
\sup _{m} \sum_{n \in \mathbf{Z}}|k(m+l, n)-k(m, n)| \leq e(l \mu) .
$$

Let $\Omega=\mathbf{Z}^{+}$or $\mathbf{Z}$. The kernel $k$ generates an operator $\mathbf{K}$ by

$$
\mathbf{K} x(m)=\sum_{n \in \Omega} k(m, n) x_{n}, \quad m \in \Omega,
$$

which is a bounded operator acting on $l^{p}(\Omega), 1 \leq p \leq \infty$, and

$$
\|\mathbf{K} x\|_{l^{p}(\Omega)} \leq\|b\|_{L^{1}(\mathbf{R})}\|x\|_{l^{p}(\Omega)} .
$$

The collection of operators $\mathbf{K}$ for which (4.30), (4.31) holds for some $\mu>0$ is denoted by $\Phi(b, e)$.

THEOREM 4.4. Let $\mathbf{E} \subset \Phi(b, e)$ and let $\lambda \in \mathbb{C}$. If $\lambda \in \rho_{2}(\mathbf{K})$ for all $\mathbf{K} \in \mathbf{E}$ and

$$
\sup \left\|(\lambda \mathbf{I}-\mathbf{K})^{-1}\right\|_{l^{2}(\Omega)}<\infty,
$$

then $\lambda \in \rho_{\infty}(\mathbf{K})$ for all $\mathbf{K} \in \mathbf{E}$ and

$$
\sup \left\|(\lambda \mathbf{I}-\mathbf{K})^{-1}\right\|_{l \infty(\Omega)}<\infty .
$$

The proof is analogous to that of Theorem 4.1. The only difference is the fact that we cannot set $\mu=1$ in (4.30), (4.31), so we have to consider the $\mu_{n}$ for each $\mathbf{K}_{n}$ as well. We omit the details.

5. The $L^{\infty}$ Setting. We are now going to apply Theorem 4.1 to the study of Eq. (1.1) in the $L^{\infty}$ setting.

As in Section 3, we write Eq. (1.1) in $L^{\infty}\left(\mathbf{R}^{+}\right)$as

$$
x+\mathbf{B}_{\lambda \nu} \mathbf{G}_{\nu}(x)=(\mathbf{I}+\lambda \nu \mathbf{A})^{-1} y
$$

with

$$
\mathbf{B}_{\lambda \nu}=\lambda \nu(\mathbf{I}+\lambda \nu \mathbf{A})^{-1} \mathbf{A}, \quad \mathbf{G}_{\nu}(x)=(\mathbf{G}(x)-\nu x) / \nu,
$$

where $\nu>0$ is so large that $\mathbf{G}_{\nu}$ is a strong contraction. Here we use (3.17)-(3.20) to establish the boundedness of the operators $\mathbf{B}_{\lambda \nu}$ and $(\mathbf{I}+\lambda \nu \mathbf{A})^{-1}=\mathbf{I}-\mathbf{B}_{\lambda \nu}$. Now the Kolodner theory cannot be applied, since in general we do not have that

$$
\left\|\mathbf{B}_{\lambda \nu}\right\|_{L^{\infty}\left(\mathbf{R}^{+}\right)} \leq 1 .
$$

However, the solvability of (5.1) can be studied by means of Hadamard's theorem, Berger [3, (5.1.5)]. For convenience we state the version we use here. 
HADAMARD'S THEOREM. Let $X$ and $Y$ be Banach spaces. Suppose that $f \in$ $C^{1}(X, Y)$ is a local homeomorphism, and let $\varsigma(R)=\inf _{\|x\| \leq R}\left(\left\|\left[f^{\prime}(x)\right]^{-1}\right\|^{-1}\right)$. If $\int_{1}^{\infty} \varsigma(R) d R=\infty$, then $f$ is a homeomorphism of $X$ onto $Y$. In particular, if $\sup \left\{\left\|\left[f^{\prime}(x)\right]^{-1}\right\|: x \in X\right\}<\infty$, then $f$ is a homeomorphism of $X$ onto $Y$.

The derivative is taken in the sense of Fréchet. Observe that the condition $\sup \left\|\left[f^{\prime}(x)\right]^{-1}\right\|<\infty$ by itself implies (by the implicit function theorem) that $f$ is a local homeomorphism. The next lemma says that Hadamard's Theorem can indeed be applied, with $f(x)=x+\mathbf{B}_{\lambda \nu} \mathbf{G}_{\nu}(x)$.

LEMMA 5.1. Let $\lambda, \mathbf{G}$ be as in (1.1). Then there exists a constant $\gamma$ such that for all $z \in L^{\infty}\left(\mathbf{R}^{+}\right)$,

$$
\left\|\left[\mathbf{I}+\mathbf{B}_{\lambda \nu} \mathbf{G}_{\nu}^{\prime}(z)\right]^{-1}\right\|_{L^{\infty}\left(\mathbf{R}^{+}\right)} \leq \gamma .
$$

Before proving this, we note

THEOREM 5.2. Let $\lambda, \mathbf{G}$ be as in (1.1). Then the equation $x+\lambda \mathbf{A G}(x)=y$ has a unique solution $x \in L^{\infty}\left(\mathbf{R}^{+}\right)$for every $y \in L^{\infty}\left(\mathbf{R}^{+}\right)$, and $x$ depends Lipschitz continuously on $y$ in the $L^{\infty}$ topology.

Proof. It suffices to ascertain the solvability of Eq. (5.1) for every $y \in L^{\infty}\left(\mathbf{R}^{+}\right)$. The Fréchet derivative of the operator $x \rightarrow x+\mathbf{B}_{\lambda \nu} \mathbf{G}_{\nu}(x)$ is the linear operator $\mathbf{I}+\mathbf{B}_{\lambda \nu} \mathbf{G}_{\nu}^{\prime}(x)$, where $\mathbf{G}_{\nu}^{\prime}(x)$ is the operator defined by (3.7). Now Lemma 5.1 tells us that the Fréchet derivatives have bounded inverses, uniformly in $x \in L^{\infty}\left(\mathbf{R}^{+}\right)$. Then Hadamard's theorem gives us the unique solvability of Eq. (5.1). Moreover, if $x^{i}$ corresponds to $y^{i}$ in Eq. (5.1), then by a standard argument, cf. Berger [3, (5.1.5)], we obtain that

$$
\left\|x^{2}-x^{1}\right\|_{L^{\infty}\left(\mathbf{R}^{+}\right)} \leq c\left\|y^{1}-y^{2}\right\|_{L^{\infty}\left(\mathbf{R}^{+}\right)},
$$

where

$$
c=\gamma\left\|[\mathbf{I}+\lambda \nu \mathbf{A}]^{-1}\right\|_{L^{\infty}\left(\mathbf{R}^{+}\right)} .
$$

Inequality (5.3) establishes the Lipschitz continuous dependence of $x$ on $y$.

Proof of Lemma 5.1. We want to apply Theorem 4.1, so we need to show that our operators $\mathbf{B}_{\lambda \nu} \mathbf{G}_{\nu}^{\prime}(z)$ form a subset $\mathbf{E}$ of some $\mathbf{F}(b, e)$. Note that

$$
\left[\mathbf{B}_{\lambda \nu} \mathbf{G}_{\nu}^{\prime}(z) x\right](t)=\int_{\mathbf{R}^{+}} k_{z}(t, s) x(s) d s
$$

where

$$
k_{z}(t, s)=b_{\lambda \nu}(t-s)\left(g^{\prime}(s, z(s))-\nu\right) / \nu,
$$

and thus, since $\mathrm{G}_{\nu}^{\prime}(z)$ is a contraction,

$$
\left|k_{z}(t, s)\right| \leq\left|b_{\lambda \nu}(t-s)\right|
$$

and also

$$
\sup _{t} \int_{-\infty}^{\infty}\left|k_{z}(t+h, s)-k_{z}(t, s)\right| d s \leq \int_{-\infty}^{\infty}\left|b_{\lambda \nu}(h+t)-b_{\lambda \nu}(t)\right| d t .
$$

We may denote the right-hand side of (5.7) by $e\left(\lambda \nu h^{\alpha}\right)$, where $e$ is independent of $\lambda, \nu$ and $h$. The inequalities (5.6), (5.7) show that the set $\mathbf{E}$,

$$
\mathbf{E}=\left\{\mathbf{B}_{\lambda \nu} \mathbf{G}_{\nu}^{\prime}(z): z \in L^{\infty}\left(\mathbf{R}^{+}\right), \lambda \in \mathbb{C},|\arg \lambda| \leq(1-\alpha) \pi / 2\right\}
$$


is a subset of $\mathbf{F}\left(\left|b_{\lambda \nu}\right|, e\right)$. Now Corollary 3.3 tells us that

$$
\sup _{\mathbf{K} \in \mathbf{E}}\left\|(\mathbf{I}+\mathbf{K})^{-1}\right\|_{L^{2}\left(\mathbf{R}^{+}\right)}<\infty
$$

and so Theorem 4.1 allows us to conclude that

$$
\sup _{\mathbf{K} \in \mathbf{E}}\left\|(\mathbf{I}+\mathbf{K})^{-1}\right\|_{L^{\infty}\left(\mathbf{R}^{+}\right)}<\infty .
$$

The application of Theorem 4.1 is justified by (3.19)-(3.20). So the lemma is proved.

The beauty of the above approach is that it goes through for the discrete Galerkin equations in exactly the same manner, using the analogue, Theorem 4.4, of Theorem 4.1. First we need the discrete analogue of (3.16)-(3.20), embodied in the following lemma.

LEMMA 5.3. There exists a constant $\gamma$ such that for all $\mu \in \mathbb{C}$, with $|\arg \mu| \leq(1-\alpha) \pi / 2$, there holds

$$
\left\|\mu(L+\mu A)^{-1} A\right\|_{l \infty} \leq \gamma .
$$

In particular,

$$
\mu(L+\mu A)^{-1} A=\left[\begin{array}{ccccc}
b_{0} & & & \\
b_{1} & b_{0} & & \\
b_{2} & b_{1} & b_{0} & \\
\vdots & \vdots & \vdots & \ddots
\end{array}\right]
$$

where the $b_{i} \in \mathbb{C}^{(P+1) \times(P+1)}$ satisfy

$$
\left\|b_{n}\right\| \leq c\left(\left[1+|\mu| n^{\alpha}\right]^{-1}-\left[1+|\mu|(n+1)^{\alpha}\right]^{-1}\right) \text { for all } n
$$

and

$$
\sum_{i=0}^{\infty}\left\|b_{n+i}-b_{i}\right\| \leq c_{1}|\mu| n^{\alpha} \quad \text { for all } n
$$

where $c$ and $c_{1}$ are constants independent of $\mu$ and $n$.

The proof is given in the appendix and for small $|\mu|$ relies on some results of de Bruijn and Erdös [6], compare Bakke and Jackiewicz [2]. For $|\mu|$ bounded away from zero, these results are derived from asymptotic results about the $b_{i}$, uniformly in $\mu$, using some results of Luxemburg [19] and the author [8].

We now prove the discrete analogue of Lemma 5.1. Let $B_{\mu}$ denote

$$
B_{\mu}=\mu(I+\mu M A M)^{-1} M A M
$$

with $M=L^{-1 / 2}$, and let

$$
H_{\nu}^{\prime}(z)=\nu^{-1}\left(G^{\prime}(z)-\nu I\right)
$$

where $\nu>0$ is chosen such that $\left\|H_{\nu}^{\prime}(z)\right\|_{l \infty}<1$. 
LEMMA 5.4. For $\mathrm{G}$ as in (1.1), there exists a constant $\gamma$ such that for all $z \in l^{\infty}$ and for all $\mu \in \mathbb{C},|\arg \mu| \leq(1-\alpha) \pi / 2$, one has

$$
\left\|\left[I+B_{\nu \mu} H_{\nu}^{\prime}(z)\right]^{-1}\right\|_{l \infty} \leq \gamma .
$$

Proof. In Lemma 5.3 we stated that $B_{\nu \mu} \in \Phi(b, e)$ for an $L^{1}$ function $b$, viz.,

$$
b(t)=c t^{\alpha-1}\left[1+t^{\alpha}\right]^{-2}
$$

and $e(t)=c_{1} t^{\alpha}$. In (4.30) and (4.31), $\mu b(\mu t)$ and $e(l \mu)$ should be replaced by $\mu^{1 / \alpha} b\left(\mu^{1 / \alpha} t\right)$ and $e\left(\mu^{1 / \alpha} l\right)$. So, we are considering the subset $\mathbf{E}$ of $\Phi(b, e)$,

$$
\mathbf{E}=\left\{B_{\nu \mu} H_{\nu}^{\prime}(z): z \in l^{\infty}, \mu \in \mathbb{C},|\arg \mu| \leq(1-\alpha) \pi / 2\right\},
$$

and since Corollary 3.6 tells us that

$$
\sup \left\|\left[I+B_{\nu \mu} H_{\nu}^{\prime}(z)\right]^{-1}\right\|_{l^{2}}<\infty,
$$

where the sup is over all operators in $\mathbf{E}$, Theorem 4.4 lets us conclude that the above inequality holds for the $l^{\infty}$ norm as well.

We now have the existence of solutions of the Galerkin equations, again by an application of Hadamard's theorem.

THEOREM 5.5. The Galerkin equations

$$
L r_{h} x_{h}+\lambda h^{\alpha} A G\left(r_{h} x_{h}\right)=L r_{h} y
$$

with $|\arg \lambda| \leq(1-\alpha) \pi / 2$ and $h>0$ have a unique solution $r_{h} x_{h} \in l^{\infty}$ for every $r_{h} y \in l^{\infty}$, which depends Lipschitz continuously on $r_{h} y$ in the $l^{\infty}$-topology, uniformly in $\lambda$ and $h$ as above.

The proof closely follows that of Theorem 5.2 and is omitted.

The above theorem describes a sort of (numerical) stability. In the next section we consider the dependence of $x_{h}$ on $x$, i.e., the stability of our method as an approximate projection method. Error estimates will follow from this.

6. Stability and Error Estimates in the $L^{\infty}$ Setting. We first consider the dependence of $x_{h}$ on $x$. We phrase the result in a slightly more general form, with an eye towards error estimation. Recall definition (1.12) of $C^{0}\left(\mathbf{R}^{+}\right)$.

THEOREM 6.1. Let $x^{1}, x^{2} \in C^{0}\left(\mathbf{R}^{+}\right)$, with $x^{2} \in D_{\mathbf{A}}$, and let $y=x^{1}+\lambda \mathbf{A} x^{2}$. Then for $|\arg \lambda| \leq(1-\alpha) \pi / 2$ and all $h>0$, the Galerkin equations

$$
L r_{h} x_{h}+\lambda h^{\alpha} A G\left(r_{h} x_{h}\right)=L r_{h} y
$$

have a unique solution $x_{h} \in S_{p}(h)$ which depends Lipschitz continuously on $x^{1}, x^{2}$ in the $L^{\infty}$-topology, uniformly in $h$, i.e., if $x_{h}^{1}$ is the solution for $y^{1}=x^{1}+\lambda \mathbf{A} x^{2}$ and $x_{h}^{3}$ is the solution for $y^{3}=x^{3}+\lambda \mathbf{A} x^{4}$, then

$$
\left\|x_{h}^{1}-x_{h}^{3}\right\|_{L^{\infty}\left(\mathbf{R}^{+}\right)} \leq c\left\{\left\|x^{1}-x^{3}\right\|_{L^{\infty}\left(\mathbf{R}^{+}\right)}+\left\|x^{2}-x^{4}\right\|_{L^{\infty}\left(\mathbf{R}^{+}\right)}\right\}
$$

with $c$ independent of $h, \lambda$.

Corollary 6.2. Let $x \in C^{0}\left(\mathbf{R}^{+}\right)$, with $\mathbf{G}(x) \in D_{\mathbf{A}}$, and let $y=x+\lambda \mathbf{A G}(x)$. Then the Galerkin equations

$$
L r_{h} x_{h}+\lambda h^{\alpha} A G\left(r_{h} x_{h}\right)=L r_{h} y
$$


define a Lipschitz continuous map

$$
C^{0}\left(\mathbf{R}^{+}\right) \ni x \rightarrow x_{h} \in S_{p}(h)
$$

uniformly in $h>0$. Moreover, this holds even if $\mathbf{G}(x) \notin D_{\mathbf{A}}$.

Proof of Theorem 6.1. If $x^{1} \in C^{0}\left(\mathbf{R}^{+}\right)$and $x^{2}=\mathbf{G}(x) \in D_{\mathbf{A}}$, then $x^{1}+\lambda \mathbf{A} x^{2} \in$ $C^{0}\left(\mathbf{R}^{+}\right)$, and Theorem 5.5 gives us the existence of the solution $x_{h}^{1} \in S_{p}(h)$ of the equation

$$
L r_{h} x_{h}^{1}+\lambda h^{\alpha} A G\left(r_{h} x_{h}^{1}\right)=L r_{h}\left(x^{1}+\lambda \mathbf{A} x^{2}\right) .
$$

Let $r_{h} x_{h}^{3}$ be the solution for the right-hand side $L r_{h}\left(x^{3}+\lambda \mathbf{A} x^{4}\right)$. Now define $y_{h}:[0,1] \rightarrow l^{\infty}$ by

$$
y_{h}(t)=t L r_{h}\left(x^{1}+\lambda \mathbf{A} x^{2}\right)+(1-t) L r_{h}\left(x^{3}+\lambda \mathbf{A} x^{4}\right),
$$

and let $x_{h}(t)$ be the solution of

$$
L r_{h} x_{h}(t)+\lambda h^{\alpha} A G\left(r_{h} x_{h}(t)\right)=y_{h}(t) .
$$

By means of the Kolodner argument (to eliminate the unbounded operator $A$ ) and the standard reasoning in connection with Hadamard's theorem, we then see that $x_{h}(t)$ is differentiable with respect to $t$, and that

$$
\begin{aligned}
& r_{h}\left(x_{h}(1)-x_{h}(0)\right) \\
& \quad=\int_{0}^{1}\left[L+\lambda h^{\alpha} A G^{\prime}\left(r_{h} x_{h}(t)\right)\right]^{-1} L r_{h}\left[\left(x^{1}-x^{3}\right)+\lambda \mathbf{A}\left(x^{2}-x^{4}\right)\right] d t .
\end{aligned}
$$

From Lemma 5.4 we know that (after the usual manipulation following Kolodner)

$$
\sup \left\|\left[L+\lambda h^{\alpha} A G^{\prime}\left(r_{h} z_{h}\right)\right]^{-1}\right\|_{l \infty}<\infty
$$

where the supremum is over all $\lambda, h$ and $z_{h}$. So the question is whether

$$
T=\lambda\left[L+\lambda h^{\alpha} A G^{\prime}\left(r_{h} z_{h}\right)\right]^{-1} L r_{h} \mathbf{A}
$$

as a mapping from $L^{\infty}\left(\mathbf{R}^{+}\right)$into $l^{\infty}$ is bounded as well.

So let $y \in L^{\infty}\left(\mathbf{R}^{+}\right)$. Similar to [10, Lemma 2.6], it can be shown that there exists a $\tilde{\psi}_{h} \in S_{p}(h)$ and $e_{h} \in l^{\infty}$ with

$$
L r_{h} \mathbf{A} y=h^{\alpha} A r_{h} \tilde{\psi}_{h}+h^{\alpha} e_{h}
$$

and

$$
\left\|\tilde{\psi}_{h}\right\|_{L^{\infty}\left(\mathbf{R}^{+}\right)}+\left\|e_{h}\right\|_{l^{\infty}} \leq c\|y\|_{L^{\infty}\left(\mathbf{R}^{+}\right)} \text {. }
$$

Since $A^{-1}: l^{\infty} \rightarrow l^{\infty}$ is bounded [10, Lemma 4.7], there exists a $\psi_{h} \in S_{p}(h)$ such that

$$
L r_{h} \mathbf{A} y=h^{\alpha} A r_{h} \psi_{h}
$$

and

$$
\left\|\psi_{h}\right\|_{L^{\infty}\left(\mathbf{R}^{+}\right)} \leq c_{1}\|y\|_{L^{\infty}\left(\mathbf{R}^{+}\right)} .
$$


(In effect, this says that the Galerkin methods under consideration, when applied to the Abel-type integral equation of the first kind on $(0, \infty)$

$$
\int_{0}^{t}(t-s)^{\alpha-1} x(s) d s=y(t), \quad t>0
$$

is stable as a projection method. In [10] this was shown only for a finite interval.)

Consequently, from (6.4)-(6.5) we get that

$$
\begin{aligned}
T y & =\lambda h^{\alpha}\left[L+\lambda h^{\alpha} A G^{\prime}\left(r_{h} z_{h}\right)\right]^{-1} A r_{h} \psi_{h} \\
& =\left\{I-\left[L+\lambda h^{\alpha} A G^{\prime}\left(r_{h} z_{h}\right)\right]^{-1} L\right\}\left[G^{\prime}\left(r_{h} z_{h}\right)\right]^{-1} r_{h} \psi_{h},
\end{aligned}
$$

and since

$$
\left\|\left[G^{\prime}\left(r_{h} z_{h}\right)\right]^{-1}\right\| \leq \delta^{-1},
$$

together with Lemma 5.4, we obtain

$$
\|T y\|_{l \infty} \leq c\left\|r_{h} \psi_{h}\right\|_{l \infty} \leq c_{1}\|y\|_{L^{\infty}\left(\mathbf{R}^{+}\right)} .
$$

Thus, $T: L^{\infty}\left(\mathbf{R}^{+}\right) \rightarrow l^{\infty}$ is bounded, uniformly in $h>0$. Since, evidently, $x_{h}(1)=$ $x_{h}^{1}$ and $x_{h}(0)=x_{h}^{3}$, we then obtain the result (6.1) from (6.2), (6.3).

The error estimates for the Galerkin method in the $L^{\infty}$ setting now follow easily.

The Galerkin approximation $x_{h}$ to $x$ satisfies

$$
L r_{h} x_{h}+\lambda h^{\alpha} A G\left(r_{h} x_{h}\right)=L r_{h}[x+\lambda \mathbf{A G}(x)],
$$

whereas $x$ satisfies, assuming $x \in C^{0}\left(\mathbf{R}^{+}\right)$,

$$
L r_{h} x+\lambda h^{\alpha} A G\left(r_{h} x\right)=L r_{h}\left[x+\lambda \mathbf{A} p_{h} r_{h} \mathbf{G}(x)\right] .
$$

Equation (6.7) holds by virtue of the construction of $A$. Now Theorem 6.1 tells us that

$$
\left\|x_{h}-p_{h} r_{h} x\right\|_{L^{\infty}\left(\mathbf{R}^{+}\right)} \leq c\left\|\mathbf{G}(x)-p_{h} r_{h} \mathbf{G}(x)\right\|_{L^{\infty}\left(\mathbf{R}^{+}\right)},
$$

and the standard projection-method argument then gives that

$$
\left\|x_{h}-p_{h} r_{h} x\right\|_{L^{\infty}\left(\mathbf{R}^{+}\right)} \leq c_{1} \inf \|\mathbf{G}(x)-\psi\|_{L^{\infty}\left(\mathbf{R}^{+}\right)}
$$

where the infimum is over all $\psi \in S_{P}(h)$. We have thus proven

THEOREM 6.3. Under the same conditions as in Theorem 6.1, the Galerkin approximations $x_{h}$ to $x$ satisfy

$$
\left\|x_{h}-p_{h} r_{h} x\right\|_{L^{\infty}\left(\mathbf{R}^{+}\right)} \leq c \inf \left\{\|\mathbf{G}(x)-\psi\|_{L^{\infty}\left(\mathbf{R}^{+}\right)}: \psi \in S_{P}(h)\right\} .
$$

COROLlaRY 6.4. If $\mathbf{G}(x) \in C^{P+1}\left(\mathbf{R}^{+}\right)$, then

$$
\left\|x_{h}-p_{h} r_{h} x\right\|_{L^{\infty}\left(\mathbf{R}^{+}\right)} \leq \operatorname{ch}^{P+1}\|\mathbf{G}(x)\|_{C^{P+1}} .
$$

Remark. If $x$ itself is not smooth, then these estimates merely say that $r_{h} x_{h}$ (the sampled values of $x_{h}$ ) are good approximations to $r_{h} x$ (the values of the function we want to find). No conclusions about the global error $\left\|x_{h}-x\right\|_{L^{\infty}\left(\mathbf{R}^{+}\right)}$can be drawn.

7. Numerical Experiments. We present some numerical results for the equation

$$
x(t)+\lambda \int_{0}^{t}(t-s)^{-1 / 2} \frac{x(s)}{1-\theta x(s)} d s=y(t), \quad t>0
$$


which arises in the singular perturbation analysis of an electrothermal rod atomizer, Paveri-Fontana and Rigacci [22]. Here $\lambda>0$ and $\theta \in[0,1)$.

The nonlinear term in (7.1) is not really covered by our analysis. However, if the solution $x(t)$ is such that $0 \leq x(t) \leq 1$, say, then a posteriori the nonlinearity belongs to the type described in (1.1). For numerical purposes, we replace the nonlinearity by

$$
g(s, x)= \begin{cases}x, & x<0 \\ \frac{x}{1-\theta x}, & 0 \leq x \leq 1 \\ \frac{x-\theta}{(1-\theta)^{2}}, & x>1\end{cases}
$$

and this does satisfy condition (1.2).

The analysis of Eq. (1.1), or (7.1), assumes that $g(t, x(t))$ and $y(t)$ are smooth functions of $t$. These are actually contradictory assumptions, and so we must modify (7.1) as follows. Assuming that $y(t)$ has an asymptotic expansion in powers of $t^{1 / 2}$ near $t=0$, it follows that $x(t)$ and $g(t, x(t))$ behave likewise, see Brunner [4]. As in [11], there exist numbers $z_{i}$ dependent on the scaling parameter $\kappa$ such that

$$
g(t, x(t))=\sum_{i=0}^{2 P+1} z_{i} \frac{(\kappa t)^{i / 2}}{(1+\kappa t)^{(i+3) / 2}}+\cdots, \quad t \rightarrow 0
$$

It is now a nice exercise to check that once the $z_{i}$ are known, we may modify Eq. (1.1) as

$$
\begin{aligned}
x(t) & +\lambda \int_{0}^{t}(t-s)^{-1 / 2}\left\{g(s, x(s))-\sum z_{i} \frac{(\kappa s)^{i / 2}}{(1+\kappa s)^{(i+3) / 2}}\right\} d s \\
& =y(t)-\lambda \kappa^{1 / 2} \sum z_{i} B\left(\frac{i}{2}, 1\right) \frac{(\kappa t)^{(i+1) / 2}}{(1+\kappa t)^{i / 2+1}}
\end{aligned}
$$

with the summations as in (7.2). It now follows from Corollary 6.4 that the Galerkin method (2.9) applied to Eq. (7.3) yields $O\left(h^{P+1}\right)$ convergence. In practice, the $z_{i}$ must be approximated as well, e.g., as in [11].

We show some results of the Galerkin method for Eq. (7.3) for various $\lambda, \theta$ and $y(t) \equiv 1$. For $\theta=0$ the solution is known, $x(t)=E_{\alpha}\left(-\lambda \Gamma(\alpha) t^{\alpha}\right)$, with $\alpha=\frac{1}{2}$. See (3.17)-(3.18). We use the Galerkin method (2.9), and for the parameters $u_{q}$ we choose the Gauss points for the interval $[0,1]$. We obtain approximations at the knots $i h$ by Lagrange interpolation of degree $2 P+1$ on the computed samples $r_{h} x_{h}$ for the intervals $[(i-1) h,(i+1) h]$. This (probably) results in some averaging of the approximation errors, and gives better results than setting $u_{P}=1$, e.g., as in the choice described following (7.4). Interpolation works well away from 0. To obtain good results near 0 , one would have to interpolate the approximately smooth 
function

$$
x_{h}(t)-\sum_{i=0}^{2 P+1} x_{i}^{h} \frac{(\kappa t)^{i / 2}}{(1+\kappa t)^{(i+3) / 2}},
$$

cf. (7.2), and then adding the correction term. We have not done so here.

We compare the results with the collocation method for Eq. (7.3),

$$
\left\{\begin{array}{l}
r_{h} x_{h}+r_{h} \mathbf{A} p_{h} r_{h} \mathbf{G}_{h}\left(x_{h}\right)=r_{h} y, \\
x_{k} \in S_{P}(h)
\end{array}\right.
$$

as described by Brunner [4]. See also [11]. Here $\mathbf{G}_{h}$ reflects the fact that the $z_{i}$ in (7.3) will depend on $h$, in general. For the parameters $u_{q}$ we choose the abscissas $\eta_{l}, l=0,1, \ldots, P$, of the interpolatory quadrature rule for the integral

$$
\int_{0}^{1} \nu(\tau)(1-\tau)^{-1 / 2} d \tau
$$

which is exact for $\nu(\tau)=\tau^{i}, i=0,1, \ldots, 2 P$, as given by te Riele [24, Table 1]. This choice appears to give the best results.

For the case $\lambda=1, \theta=0$ the results of the Galerkin method and collocation method are roughly equivalent: the Galerkin method is slightly more accurate at points far away from 0 , while the collocation method tends to be more accurate close to 0 . This holds for both $P=2$ and $P=3$.

We also give some results for Eq. (7.3) with $\lambda=0.1$ and $\theta=0.5$. Since the solution is not known, we present the results in Table 3 together with some asymptotic information. We also give the results of $\lambda=1, \theta=0$ in the same format, for comparison purposes (Table 4). The nonlinearity does not appear to affect the performance of the Galerkin and collocation method. Again, both methods appear to be comparable.

If we try to solve (7.1) as is, e.g., for $\lambda=1, \theta=0$, then the results are not very good for the collocation method $(P=3, h=0.0125)$, the error being $.272(-7)$ at 10.0. However, for the Galerkin method the error is .218(-13) already for stepsize $h=0.1$. The effect of interpolation is marginal: the error at the nearest interpolation point is $.177(-12)$. However, for both methods the error is about $.3(-3)$ close to 0 . See Table 5 . We may thus conclude that the long-range stability of the Galerkin method appears to have practical consequences as well.

As a final comment, we discuss the computational effort involved. Once the system matrices and right-hand sides have been determined, the cost of solving the system of equations is exactly the same for both the Galerkin method and the collocation method. The cost of computing the right-hand side for the Galerkin method is negligible. However, the cost of computing the system matrix for the Galerkin method is a lot higher than that for the collocation method. It thus appears that the collocation method is easier to apply. The advantage of the Galerkin method is largely theoretical: we can prove its stability for arbitrarily long intervals. For the collocation method this is next to impossible (compare with the similar situation for Abel-type integral equations of the first kind, [10]). It can be argued that the long-interval stability of the Galerkin method is the reason for the better long-range behavior of the method. 
TABLE 1a

Collocation for (7.3). $\lambda=1, \theta=0, P=2, \kappa=5$.

\begin{tabular}{|c|c|c|c|c|c|c|c|}
\hline$h$ & 0.1 & & 0.05 & & 0.025 & & 0.0125 \\
\hline $\begin{array}{l}t \\
0.1\end{array}$ & $.296(-5)$ & & $.109(-5)$ & & $.642(-7)$ & & $-.117(-8)$ \\
\hline & & 2.7 & & 17 & & 55 & \\
\hline 1.0 & $-.655(-6)$ & & $-.110(-7)$ & & $.183(-8)$ & & $.244(-9)$ \\
\hline & & 60 & & 6.0 & & 7.5 & \\
\hline 10.0 & $-.299(-7)$ & & $-.102(-8)$ & & $.507(-11)$ & & $.119(-11)$ \\
\hline $\max$ & $.474(-4)$ & 29 & $-.257(-4)$ & 200 & $-.384(-5)$ & 4.2 & $-.328(-6)$ \\
\hline
\end{tabular}

TABLE 1b

Galerkin for (7.3). $\lambda=1, \theta=0, P=2, \kappa=5$.

\begin{tabular}{|c|c|c|c|c|c|c|c|}
\hline$h$ & 0.1 & & 0.05 & & 0.025 & & 0.0125 \\
\hline$t$ & & & & & & & \\
\hline \multirow[t]{2}{*}{0.1} & $.987(-4)$ & & $.382(-5)$ & & $.305(-6)$ & & $.266(-7)$ \\
\hline & & 26 & & 12 & & 12 & \\
\hline \multirow[t]{2}{*}{1.0} & $-.325(-7)$ & & $-.316(-8)$ & & $-.599(-9)$ & & $-.668(-10)$ \\
\hline & $180(-8)$ & 10 & & 5.3 & & 9.0 & $210(-12)$ \\
\hline 10.0 & $.180(-8)$ & 9.1 & $.197(-9)$ & 30 & $.659(-11)$ & 31 & $.210(-12)$ \\
\hline \multirow[t]{2}{*}{$\max$} & $.987(-4)$ & & $.609(-4)$ & & $.439(-4)$ & & $.316(-4)$ \\
\hline & & 1.6 & & 1.39 & & 1.39 & \\
\hline
\end{tabular}

\section{TABLE 2a}

Collocation for (7.3). $\lambda=1, \theta=0, P=3, \kappa=5$.

\begin{tabular}{|c|c|c|c|c|c|c|c|}
\hline$h$ & 0.1 & & 0.05 & & 0.025 & & 0.0125 \\
\hline \multirow{3}{*}{0.1} & & & & & & & \\
\hline & $-.280(-7)$ & & $.376(-8)$ & & $.108(-9)$ & & $-.775(-11)$ \\
\hline & $-.192(-8)$ & 7.4 & $.893(-11)$ & 35 & $.164(-11)$ & 14 & $.143(-12)$ \\
\hline 1.0 & $-.192(-0)$ & 210 & $.080(-11)$ & 5.4 & $.107(-11)$ & 11 & \\
\hline \multirow[t]{2}{*}{10.0} & $-.931(-10)$ & & $-.138(-12)$ & & $.567(-13)$ & & $.595(-14)$ \\
\hline & $.129(-5)$ & 670 & $.132(-6)$ & 2.4 & $.890(-8)$ & 9.5 & $.130(-8)$ \\
\hline $\max$ & & 9.8 & & 15 & & 6.8 & \\
\hline
\end{tabular}

\section{TABLE $2 \mathrm{~b}$}

Galerkin for (7.3). $\lambda=1, \theta=0, P=3, \kappa=5$.

\begin{tabular}{|c|c|c|c|c|c|c|c|}
\hline \multirow{2}{*}{$t^{h}$} & \multicolumn{2}{|l|}{0.1} & \multicolumn{2}{|l|}{0.05} & \multicolumn{2}{|l|}{0.025} & \multirow[t]{2}{*}{0.0125} \\
\hline & & & & & & & \\
\hline 0.1 & $-.893(-5)$ & & $-.244(-9)$ & & $.705(-9)$ & & $.365(-10)$ \\
\hline 1.0 & $.369(-9)$ & & $.192(-10)$ & 3.5 & $.950(-12)$ & 19 & $.154(-12)$ \\
\hline & & 19 & $408(-12)$ & 20 & $520(-13)$ & 6.2 & $527(-13)$ \\
\hline 10.0 & ) & 2.5 & $.408(-12)$ & 7.8 & & 1.0 & $.020(10)$ \\
\hline $\max$ & $-.893(-5)$ & & $-.624(-5)$ & & $-.442(-5)$ & & $-.314(-5)$ \\
\hline
\end{tabular}


TABLE 3a

Collocation for (7.3). $\lambda=0.1, \theta=0.5, P=2, \kappa=5$.

Shown are the approximate solutions for various points and stepsizes, the differences between solutions for consecutive stepsizes, and their ratios.

\begin{tabular}{rrrrrrrr}
\multicolumn{1}{c}{$h$} & 0.1 & & 0.05 & & 0.025 & 0.0125 \\
$t$ & & & & & \\
0.1 & .893388813 & & .893387888 & & .893387727 & & .893387712 \\
& & $-.925(-6)$ & 5.8 & $-.160(-6)$ & 10.2 & $-.157(-7)$ & \\
1.0 & .740500181 & & .740500078 & & .740500081 & & .740500081 \\
& & $-.103(-6)$ & -36.1 & $.285(-8)$ & -9.0 & $-.315(-9)$ & \\
10.0 & .497373850 & & .497373836 & & .497373837 & & .497393837 \\
& & $-.138(-7)$ & -27.6 & $.501(-9)$ & -27.3 & $-.184(-10)$ &
\end{tabular}

\section{TABLE $3 \mathrm{~b}$}

Galerkin for (7.3). $\lambda=0.1, \theta=0.5, P=2, \kappa=5$.

\begin{tabular}{lrrrrrrr}
\multicolumn{1}{c}{$h$} & 0.1 & & 0.05 & & 0.025 & & 0.0125 \\
$t$ & & & & & & \\
0.1 & .89340397 & & .893388202 & & .893387751 & & .893387714 \\
& & $-.157(-4)$ & 34.8 & $-.451(-6)$ & 12.1 & $-.373(-7)$ & \\
1.0 & .740500076 & & .740500079 & & .740500081 & & .740500081 \\
& & $.328(-8)$ & 2.3 & $.143(-8)$ & 6.7 & $.214(-9)$ & \\
10.0 & .497373841 & & .497373837 & & .497373837 & & .497373837 \\
& & $-.418(-8)$ & 20.0 & $-.209(-9)$ & 30.9 & $-.674(-11)$ &
\end{tabular}

\section{TABLE 4a}

Collocation for (7.3). $\lambda=1, \theta=0, P=2, \kappa=5$.

\begin{tabular}{lrrrrrrr}
\multicolumn{1}{c}{$h$} & 0.1 & & 0.05 & & 0.025 & & 0.0125 \\
$t$ & & & & & & \\
0.1 & .585943790 & & .585941925 & & .585940895 & & .585940829 \\
& & $-.186(-5)$ & 1.8 & $-.103(-5)$ & 15.8 & $-.653(-7)$ & \\
1.0 & .282058521 & & .282059165 & & .282059178 & & .282059176 \\
& & $.644(-6)$ & 50.1 & $.129(-7)$ & -8.1 & $-.159(-8)$ & \\
10.0 & .099127365 & & .099127395 & & .099127395 & & .099127395 \\
& & $.289(-7)$ & 28.3 & $.102(-8)$ & -263 & $-.388(-11)$ &
\end{tabular}

TABLE 4b

Galerkin for (7.3). $\lambda=1, \theta=0, P=2, \kappa=5$.

\begin{tabular}{lrrrrrrr}
\multicolumn{1}{c}{$h$} & 0.1 & & 0.05 & 0.025 & & 0.0125 \\
$t$ & & & & & & \\
0.1 & .586039525 & & .585944651 & & .585941136 & & .585940857 \\
& & $-.949(-4)$ & 27.0 & $-.351(-5)$ & 12.6 & $-.279(-6)$ & \\
1.0 & .282059144 & & .282059173 & & .282059176 & & .282059176 \\
& & $.294(-7)$ & 11.5 & $.256(-8)$ & 4.8 & $.533(-9)$ & \\
10.0 & .099127397 & & .099127395 & & .099127395 & & .099127395 \\
& & $-.160(-8)$ & 8.4 & $-.191(-9)$ & 29.9 & $-.638(-11)$ &
\end{tabular}




\section{TABLE 5}

Galerkin for (7.1). $\lambda=1, \theta=0, P=3$.

Shown are the errors in the computed solution at the meshpoints $i h$ obtained by interpolation on the eight sample points in the interval $((i-1) h,(i+1) h)$ for various points and stepsizes. The maximum errors shown are those for the Galerkin scheme proper. Also shown are the ratios of errors for consecutive stepsizes when the errors are substantially smaller than machine precision (about $10^{-13}$ ).

\begin{tabular}{rrrrrrrr}
\multicolumn{1}{c}{$h$} & 0.1 & & 0.05 & & 0.025 & & 0.0125 \\
$t$ & & & & & & & \\
0.1 & $.845(-3)$ & & $-.302(-5)$ & & $-.203(-7)$ & & $-.466(-9)$ \\
1.0 & $-.688(-9)$ & & $-.238(-10)$ & & $-.779(-12)$ & & $-.108(-12)$ \\
& & 30 & & 31 & & 7.2 & \\
10.0 & $.218(-13)$ & & $.525(-13)$ & & $.586(-13)$ & & $-.654(-13)$ \\
$\max$ & $-.845(-3)$ & & $.593(-2)$ & & $.419(-2)$ & & $.296(-2)$ \\
& & 1.42 & & 1.42 & & 1.42 &
\end{tabular}

Shown are the errors in the computed solution for various points and stepsizes. Also shown are the ratios of errors for consecutive stepsizes.

Appendix. Here we set out to prove Lemma 5.3. It is helpful to introduce the class $\Sigma(P, \beta)$ of sequences of $P \times P$ matrices that tend to zero at a certain rate:

$$
\Sigma(P, \beta)=\left\{\left\{a_{n}\right\}_{n \geq 0}: a_{n} \in \mathbb{C}^{P \times P}, \lim _{n \rightarrow \infty} n^{-\beta} a_{n} \text { exists }\right\} .
$$

It is convenient to write $\hat{a}(\xi) \in \Sigma(P, \beta)$ when $\hat{a}(\xi)=\sum_{n=0}^{\infty} a_{n} \xi^{n}$ and $\left\{a_{n}\right\}_{n} \in$ $\Sigma(P, \beta)$. Confusion should not arise because of this. Note that $\Sigma(P, \beta) \subset \Sigma(P, \gamma)$ for $\beta<\gamma$. We have the following results.

LEMMA A.1 (a) (Luxemburg [19]). Let $\beta, \gamma<-1$. If $A \in \Sigma(P, \beta)$ and $B \in$ $\Sigma(P, \gamma)$, then $\left\{\sum_{i=0}^{n} A_{n-i} B_{i}\right\}_{n} \in \Sigma(P, \delta)$, where $\delta=\max (\beta, \gamma)$.

(b) (Luxemburg [19], Eggermont [8]). If $A \in \Sigma(P, \beta)$ for some $\beta<-1$, and $\hat{A}(\xi)$ is nonsingular for all $|\xi| \leq 1$, then $\hat{V}(\xi)=[\hat{A}(\xi)]^{-1} \in \Sigma(P, \beta)$, and if $\lim _{n \rightarrow \infty} n^{-\beta} A_{n}=\Gamma$, then $\lim _{n \rightarrow \infty} n^{-\beta} V_{n}=-[\hat{A}(1)]^{-1} \Gamma[\hat{A}(1)]^{-1}$.

Remark. In part (b) of the lemma the matrix case $P>1$ reduces to the scalar case $P=1$ by means of Cramer's rule.

We now state some salient facts about the Galerkin matrix $A$. We let

$$
\hat{A}(\xi)=\sum_{n=0}^{\infty} L_{0}^{-1 / 2} A_{n} L_{0}^{-1 / 2} \xi^{n}, \quad|\xi|<1,
$$

where $A_{i}$ is given by $(2.10)$ and the discussion following it, and $L_{0} \in \mathbb{R}^{(P+1) \times(P+1)}$ is the diagonal block of $L$.

LEMMA A.2. There exists a nonsingular matrix $U \in \mathbf{R}^{(P+1) \times(P+1)}$ such that for all $|\xi|<1$

$$
U \hat{A}(\xi) U^{-1}=\hat{\Theta}(\xi) \hat{C}(\xi)
$$

where $C \in \Sigma(P+1,-\alpha-1)$ and

$$
\hat{\Theta}(\xi)=\left(\begin{array}{cc}
\hat{\theta}(\xi) & 0 \\
0 & I
\end{array}\right)
$$


in which

$$
\hat{\theta}(\xi)=\left(\begin{array}{cc}
(1-\xi)^{-\alpha} \hat{\psi}(\xi) & -\gamma(1-\xi)^{1-\alpha} \\
\gamma(1-\xi)^{1-\alpha} & 1-\gamma^{2}(1-\xi)^{2-\alpha} / \hat{\psi}(\xi)
\end{array}\right) .
$$

Here, $\gamma=\frac{1}{12} \alpha(\alpha+1) \Gamma(\alpha-1)$,

$$
(1-\xi)^{-\alpha} \hat{\psi}(\xi)=\sum_{n=0}^{\infty}\left\{(n+1)^{\alpha+1}-2 n^{\alpha+1}+(n-1)_{+}^{\alpha+1}\right\} \xi^{n}
$$

$\psi \in \Sigma(1,-\alpha-1)$, and $\hat{\psi}(\xi) \neq 0$ for all $|\xi| \leq 1$. Moreover, for all $\mu \in \mathbb{C},|\arg \mu| \leq$ $(1-\alpha) \pi / 2$ there holds

$$
\operatorname{Re}(x, \mu \hat{A}(\xi) x)_{\mathbb{C}^{P+1}} \geq 0, \quad|\xi|<1, x \in \mathbb{C}^{P+1}
$$

and $[\hat{A}(\xi)]^{-1} \in \Sigma(P+1,-\alpha-1)$.

Proof. We begin by showing (A.6). Let $|\xi|<1$ and $x \in \mathbb{C}^{P+1}$. Define $y \in l^{2}$ by $y^{*}=\left(y_{0}^{*}\left|y_{1}^{*}\right| \cdots\right)$ in which $y_{i}=\xi^{i} x$. Then

$$
(x, \hat{A}(\xi) x)_{\mathbb{C}^{P+1}}=(y, A y)_{l^{2}},
$$

and (A.6) follows from Lemma 3.4.

Now to (A.3). Let $U\left(L_{0}\right)^{1 / 2}$ be the transformation of $\mathbb{C}^{P+1}$ that corresponds to the change of basis $l_{q}(x), q=0,1, \ldots, P$, for $\mathbf{P}_{p}$ to the basis $p_{q}(x), q=0,1, \ldots, P$, where $p_{q}(x)=w_{q}^{1 / 2} P_{q}(2 x-1)$ is the normalized, shifted Legendre polynomial of degree $q$. We denote the transformed $A_{i}$ again by $A_{i}$. Then

$$
\left(A_{i}\right)_{q r}=\int_{0}^{1} p_{q}(x) \int_{0}^{1}(i+x-y)_{+}^{\alpha-1} p_{r}(y) d y .
$$

Straightforward calculations show that

$$
\left(A_{i}\right)_{0,0}=\left[(i+2)^{\alpha+1}-2 i^{\alpha+1}+(i-1)_{+}^{\alpha+1}\right] / \alpha(\alpha+1),
$$

cf. (A.5), and it is shown in [9, Lemma 4.6] that

$$
\alpha(\alpha+1) \sum_{i=0}^{\infty}\left(A_{i}\right)_{00} \xi^{i}=(1-\xi)^{-\alpha} \hat{\psi}(\xi), \quad|\xi|<1,
$$

with $\psi \in \Sigma(1,-\alpha-1)$ and $\hat{\psi}(\xi) \neq 0$ for all $|\xi| \leq 1$, and thus also that $[\hat{\psi}(\xi)]^{-1} \in$ $\Sigma(1,-\alpha-1)$, by Lemma A.1.

Writing $(i+x-y)_{+}^{\alpha-1}=i^{\alpha-1}+(x-y) i^{\alpha-2}+O\left(i^{\alpha-3}\right)$ for $i \rightarrow \infty$, we get

$$
A_{i q r}=i^{\alpha-1}\left(p_{q}, 1\right)\left(p_{r}, 1\right)+i^{\alpha-2}\left\{\left(p_{q}, x-\frac{1}{2}\right)-\left(p_{r}, x-\frac{1}{2}\right)\right\}+O\left(i^{\alpha-3}\right),
$$

where $(\cdot, \cdot)$ is the $L^{2}(0,1)$ inner product. It follows from the orthogonality of the Legendre polynomials that $a_{i q r}=O\left(i^{\alpha-3}\right)$ for $(q, r) \neq(0,0),(1,0),(0,1)$. Since $\left(A_{i}\right)_{1,0} \sim \frac{1}{12} i^{\alpha-2}$, it follows that

$$
[\hat{A}(\xi)]_{1,0}=\Gamma(\alpha-1)(1-\xi)^{1-\alpha} / 12+\hat{a}(\xi)
$$

with $\hat{a} \in \Sigma(1, \alpha-3)$, and similarly for $[\hat{A}(\xi)]_{0,1}$. All this shows, since $\hat{\theta}_{11}(\xi) \in$ $\Sigma(1,-\alpha-1)$, that

$$
\hat{A}(\xi)=\alpha(\alpha+1) \hat{\Theta}(\xi)+\hat{E}(\xi)
$$


with $E \in \Sigma(P+1,-\alpha-1)$. One verifies that $\operatorname{det} \hat{\theta}(\xi)=(1-\xi)^{-\alpha} \hat{\psi}(\xi)$ never vanishes, whence

$$
[\hat{\theta}(\xi)]^{-1}=\left[\begin{array}{cc}
(1-\xi)^{\alpha} \hat{\psi}(\xi)-\gamma^{2}(1-\xi)^{2} & -\gamma(1-\xi) / \hat{\psi}(\xi) \\
\gamma(1-\xi) / \hat{\psi}(\xi) & 1
\end{array}\right]
$$

and thus by Lemma A.1, $[\hat{\theta}(\xi)]^{-1} \in \Sigma(2,-\alpha-1)$. Therefore, $[\hat{\theta}(\xi)]^{-1}$ and $[\hat{\theta}(\xi)]^{-1} \hat{E}(\xi) \in \Sigma(P+1,-\alpha-1)$. Thus,

$$
\hat{A}(\xi)=\hat{\theta}(\xi)\left[\alpha(\alpha+1) I+[\hat{\theta}(\xi)]^{-1} \hat{E}(\xi)\right]
$$

and (A.3)-(A.5) follow. Finally, as in [9], using (A.6), it can be shown that $\hat{C}(\xi)$ is nonsingular for all $|\xi| \leq 1$ and thus by Lemma A.1 also $[\hat{C}(\xi)]^{-1} \in \Sigma(P+1,-\alpha-1)$; then, finally, $[\hat{A}(\xi)]^{-1} \in \Sigma(P+1,-\alpha-1)$.

We are now ready for

Proof of Lemma 5.3 for $\mu$ bounded away from zero. Let $\mu \in \mathbb{C},|\arg \mu| \leq$ $(1-\alpha) \pi / 2$ and $|\mu| \geq r$ for some $r>0$. Note that in Lemma 5.3 the condition (5.11) is vacuously satisfied (once (5.8) holds).

It follows from (A.6) that

$$
\operatorname{Re}(x,[I+\mu \hat{A}(\xi)] x)_{\mathbb{C}^{P+1}} \geq\|x\|_{\mathbb{C}^{P+1}}^{2},
$$

and thus $[I+\mu \hat{A}(\xi)]^{-1}$ exists for all $|\xi|<1$. Now set

$$
\hat{W}(\xi)=\mu[I+\mu \hat{A}(\xi)]^{-1} \hat{A}(\xi)=\left[I+\mu^{-1} \hat{V}(\xi)\right]^{-1}
$$

with

$$
\hat{V}(\xi)=[\hat{A}(\xi)]^{-1} \in \Sigma(P+1,-\alpha-1) .
$$

We thus have $V_{n} \sim \Gamma n^{-\alpha-1}$ for some $\Gamma$, and then $W_{n} \sim W_{*} n^{-\alpha-1}$ with

$$
W_{*}=\mu^{-1}\left[I+\mu^{-1} \hat{V}(1)\right]^{-1} \Gamma\left[I+\mu^{-1} \hat{V}(1)\right]^{-1} \text {. }
$$

Moreover, the asymptotic behavior holds uniformly in $\mu^{-1}$, since $\mu^{-1}$ is an element of the set

$$
\{z \in \mathbb{C}:|z| \leq r,|\arg z| \leq(1-\alpha) \pi / 2\}
$$

which is a compact set, cf. [8, p. 264]. Thus we have (5.10), since $|\mu| \geq r$ is bounded away from zero. Then also $\sum_{i=0}^{\infty}\left\|W_{n}\right\|<\infty$ uniformly in $\mu$, which is (5.8).

The proof of Lemma 5.3 for $|\mu| \leq r$ is more involved. We first consider the class of mappings $a$ of $S_{r}=\{\mu \in \mathbb{C}:|\mu| \leq r,|\arg \mu| \leq(1-\alpha) \pi / 2\}$ into the set of absolutely summable sequences of $P \times P$ matrices, i.e., $a: \mu \in S_{r} \rightarrow\left\{a_{n}(\mu)\right\}_{n} \in l^{1}$, for which there exist constants $m_{1}(a), m_{2}(a)$ such that

$$
\begin{gathered}
\|a\|_{M}=\sup _{\mu \in S_{r}} \sum_{n=0}^{\infty}\left\|a_{n}(\mu)\right\|<\infty \\
\sum_{i=0}^{\infty}\left\|a_{i-n}(\mu)-a_{i}(\mu)\right\| \leq m_{1}(a)|\mu| n^{\alpha}, \\
\left\|a_{n}(\mu)\right\| \leq m_{2}(a)\left(\left[1+|\mu| n^{\alpha}\right]^{-1}-\left[1+|\mu|(n+1)^{\alpha}\right]^{-1}\right) .
\end{gathered}
$$

In (M.2) elements with negative subscripts are defined to be zero. We denote this class of mappings by $M\left(S_{r}, P\right)$, or also just $M(P)$. In $M(P)$ convolution is defined in the usual way: $a * b(\mu)$ for $\mu \in S_{r}$ is defined by

$$
(a(\mu) * b(\mu))_{n}=\sum_{i=0}^{n} a_{n-i}(\mu) b_{i}(\mu), \quad n \geq 0 .
$$


If $a \in M(P)$, then

$$
\hat{a}(\mu, \xi)=\sum_{n=0}^{\infty} a_{n}(\mu) \xi^{n}, \quad|\xi| \leq 1
$$

and then $(a(\mu) * b(\mu))^{\curlyvee}(\xi)=\hat{a}(\mu, \xi) \hat{b}(\mu, \xi)$. If $a \in M(P)$ we also say $\hat{a}(\mu, \xi) \in M(P)$, and vice versa. We have the following results (compare Luxemburg [19]).

LEMMA A.3. If $x \in \Sigma(P,-\alpha-1)$ and $a, b \in M(P)$, then $\mu x$ and $a * b$ are both elements of $M(P)$, and

$$
\begin{aligned}
& \|a * b\|_{M} \leq\|a\|_{M}\|b\|_{M}, \\
& m_{1}(a * b) \leq\|a\|_{M} m_{1}(b), \\
& m_{2}(a * b) \leq\left[\|b\|_{M} m_{2}(a)+\|a\|_{M} m_{2}(b)\right] \cdot 2^{\alpha} .
\end{aligned}
$$

Proof. It is easily verified that the mapping $\mu \in S_{r} \rightarrow \mu x$, where $x$ is a fixed element of $\Sigma(P,-\alpha-1)$, is an element of $M\left(S_{r}, P\right)$. Obviously, $\|a(\mu) * b(\mu)\|_{l^{1}} \leq$ $\|a(\mu)\|_{l^{1}}\|a(\mu)\|_{l^{1}}$, and we get (M.1). Also, from

$$
(1-\xi)^{n}(a(\mu) * b(\mu))^{\wedge}(\xi)=\hat{a}(\mu, \xi)\left[(1-\xi)^{n} \hat{b}(\mu, \xi)\right]
$$

it follows that

$$
\sum_{i=0}^{\infty}\left\|(a(\mu) * b(\mu))_{i-n}-(a(\mu) * b(\mu))_{i}\right\| \leq\|a(\mu)\|_{l^{1}} \sum_{i=0}^{\infty}\left\|b_{i-n}(\mu)-b_{i}(\mu)\right\|,
$$

where elements with negative subscripts vanish. Thus $a(\mu) * b(\mu)$ satisfies (M.2), and the estimate for $m_{1}(a * b)$ follows.

Finally (dropping the $\mu$ dependence in the notation),

$$
\left\|(a * b)_{n}\right\| \leq \sum_{i=0}^{k}\left\|a_{i}\right\|\left\|b_{n-i}\right\|+\sum_{i=k+1}^{n}\left\|a_{i}\right\|\left\|b_{n-i}\right\|
$$

where $k=\lfloor n / 2\rfloor$, which can be estimated as

$$
\left(\sup _{j \geq n / 2}\left\|b_{j}\right\|\right)\|a\|_{l^{1}}+\left(\sup _{i \geq n / 2}\left\|a_{i}\right\|\right)\|b\|_{l^{1}} .
$$

By the properties (M.1)-(M.3) for both $a$ and $b$, the property (M.3) of $a * b$ follows, as well as the estimate for $m_{2}(a * b)$ : the factor $2^{\alpha}$ is the upper bound of $\left[1+|\mu| n^{\alpha}\right] /\left[1+|\mu| k^{\alpha}\right]$. The lemma follows.

COROLlaRY A.4. If $\alpha \in M(P)$ and $a^{* k}$ is the $k$-fold convolution of a with itself, then

$$
\begin{aligned}
& \left\|a^{* k}\right\|_{M} \leq\|a\|_{M}^{k}, \\
& m_{1}\left(a^{* k}\right) \leq m_{1}(a)\|a\|_{M}^{k-1}, \\
& m_{2}\left(a^{* k}\right) \leq m_{2}(a) k\left(2^{\alpha}\|a\|_{M}\right)^{k-1} .
\end{aligned}
$$

LEMMA A.5. If $a \in M(P)$ and $\|a\|_{M} \leq \sigma<1$, then also

$$
I-[I-\hat{a}(\mu, \xi)]^{-1} \in M(P) .
$$


Proof. Setting $\hat{b}(\mu, \xi)=I-[I-\hat{a}(\mu, \xi)]^{-1}$, we get

$$
\hat{b}(\mu, \xi)=\sum_{k=1}^{\infty}[\hat{a}(\mu, \xi)]^{k}=\sum_{k=1}^{\infty}\left[a^{* k}\right]^{\wedge}(\mu, \xi),
$$

and so $\|b(\mu)\|_{l^{1}} \leq \sum_{1}^{\infty}\|a(\mu)\|^{k} \leq \sigma(1-\sigma)^{-1}$. Then also $\hat{b}(\mu, \xi)$ satisfies (M.2) with $m_{1}(b)=m_{1}(a) /(1-\sigma)$. To prove (M.3), choose $N$ so large that $2^{\alpha} \sigma^{N} \leq \sigma$, and set $x=a^{* N}$. Then $\|x\|_{M} \leq \sigma^{N}$, and so $2^{\alpha}\|x\|_{M} \leq \sigma$. Note that by Corollary A.4 we then have $x \in M(P)$ with $m_{2}\left(x^{* k}\right) \leq m_{2}(x) k \sigma^{k-1}$. Now write $b(\mu)$ as

$$
b(\mu)=Q(\mu)+R(\mu)+Q(\mu) R(\mu)
$$

with

$$
Q(\mu)=\sum_{k=1}^{N-1} a^{* k}(\mu), \quad R(\mu)=\sum_{k=1}^{\infty} x^{* k}(\mu) .
$$

Then $Q \in M(P)$, since it is a finite linear combination of elements of $M(P)$. Since $\|x\|_{M}<1$, by what we have shown above, we have that $R$ satisfies (M.1)-(M.2). It is easily verified that $R$ satisfies (M.3) with

$$
m_{2}(R) \leq \sum_{k=1}^{\infty} m_{2}\left(x^{* k}\right) \leq m_{2}(x) /(1-\sigma)^{2} .
$$

Thus, $R \in M(P)$, and then so is $b$.

After these preliminaries (sic) we are ready to prove Lemma 5.3 for small $\mu$. We start with the scalar case $P=0$.

LEMMA A.6. Let $\hat{\phi}(\xi)=(1-\xi)^{-\alpha} \hat{\psi}(\xi)$, see (A.5). Then

$$
\mu[1+\mu \hat{\phi}(\xi)]^{-1} \hat{\phi}(\xi) \in M\left(S_{1}, P\right)
$$

Proof. First let $\mu \in \mathbf{R}, 0 \leq \mu \leq 1$. It is shown in [9], using some results of de Bruijn and Erdös [6], that $[1+\mu \hat{\phi}(\xi)]^{-1}=\sum_{i=0}^{\infty} \phi_{i} \xi^{i}$ with $\phi_{0}=(1+\mu)^{-1}$, $\phi_{i}<\phi_{i+1}<0$, and $\left|1-\phi_{0}\right|+\sum_{i=1}^{\infty}\left|\phi_{i}\right|=1$. (See also Bakke and Jackiewicz [2].) So, upon setting

$$
\hat{a}(\mu, \xi)=1-[1+\mu \hat{\phi}(\xi)]^{-1}=\mu[1+\mu \hat{\phi}(\xi)]^{-1} \hat{\phi}(\xi),
$$

we obtain $\|a(\mu)\|_{l^{1}}=1$ and $a_{i}(\mu)>a_{i+1}(\mu)>0$ for all $i \geq 0$. It follows that

$$
\sum_{i=0}^{\infty}\left|a_{i-n-1}(\mu)-a_{i}(\mu)\right|=2 \sum_{i=0}^{n} a_{i}(\mu)
$$

Since $e^{-1} \leq e^{-i / n}$ for $i=0,1, \ldots, n$, we then get that, cf. de Bruijn and Erdös [6],

$$
e^{-1} \sum_{i=0}^{n} a_{i}(\mu)<\sum_{i=0}^{n} a_{i}(\mu) e^{-i / n}<\sum_{i=0}^{\infty} a_{i}(\mu) e^{-i / n}=\hat{a}\left(\mu, e^{-1 / n}\right),
$$

and thus (M.2) follows (noting that $\hat{\psi}(1) \neq 0$ ). To show (M.3), we use the same trick, leading to

$$
\sum_{i=n}^{\infty} a_{i}(\mu)<\left(1-e^{-1}\right)^{-1} \sum_{i=0}^{\infty} a_{i}(\mu)\left(1-e^{-i / n}\right)=\left(1-e^{-1}\right)^{-1}\left[1-\hat{a}\left(\mu, e^{-1 / n}\right)\right]
$$


and so

$$
\sum_{i=n}^{\infty} a_{i}(\mu) \leq c\left[1+\mu n^{\alpha}\right]^{-1} .
$$

Now to prove (M.3), first suppose that $\mu n^{\alpha} \geq 1$. From (A.8) and the monotonicity and positivity of the $a_{i}(\mu)$ we get for all $k>0$,

$$
(k+1) a_{n+k}(\mu) \leq \sum_{i=n}^{n+k} a_{i}(\mu) \leq c\left[1+\mu n^{\alpha}\right]^{-1} .
$$

Now take $k=\left\lfloor\left(1+\mu n^{\alpha}\right) / \mu n^{\alpha-1}\right\rfloor$. Then $n \leq k \leq 2 n$, and

$$
a_{n+k}(\mu) \leq c \mu n^{\alpha-1}\left[1+\mu n^{\alpha}\right]^{-2} .
$$

Now $a_{3 n} \leq a_{n+k}$, and so

$$
a_{n}(\mu) \leq c_{1} \mu n^{\alpha-1}\left[1+\mu n^{\alpha}\right]^{-2}
$$

which is equivalent to (M.3).

Now suppose that $\mu n^{\alpha} \leq 1$. By the monotonicity of the $a_{i}(\mu)$ we get from (M.2) (already established) that $(n+1) a_{n}(\mu) \leq c \mu n^{\alpha}$, and so $a_{n}(\mu) \leq c \mu n^{\alpha-1}$. Now for $\mu n^{\alpha} \leq 1$, this implies the estimate (A.9), since $\left[1+\mu n^{\alpha}\right]^{-2} \geq 1 / 4$. So again (M.3) follows.

Thus, $\hat{a}(\mu, \zeta) \in M(P)$ for real $\mu, 0<\mu \leq 1$.

Now consider the complex case $|\mu| \leq 1,|\arg \mu| \leq(1-\alpha) \pi / 2$. Then, let $v=$ $e^{i \arg \mu}$ and set $R=[\cos (\arg \mu)]^{-1}$. One verifies that $\eta=(v-R) / R$ satisfies $|\eta|=\sin (\arg \mu) \leq \cos \alpha \pi / 2$, which is strictly less than 1 . Now do the familiar Kolodner manipulations to obtain

$$
\hat{a}(\mu, \xi)=\frac{v}{R}[1+\eta \hat{a}(R|\mu|, \xi)]^{-1} \hat{a}(R|\mu|, \xi),
$$

and so by Lemma A.5 then $\hat{a}(\mu, \xi) \in M\left(S_{1}, 1\right)$.

LEMMA A.7. Let $\hat{\Theta}(\xi)$ be as in (A.4). Then there exists an $r>0$ such that

$$
\mu[1+\mu \hat{\Theta}(\xi)]^{-1} \hat{\Theta}(\xi) \in M\left(S_{r}, P+1\right) .
$$

Proof. It suffices of course to consider $\hat{\theta}(\xi)$ instead of $\hat{\Theta}(\xi)$. Set $\hat{d}(\mu, \xi)=$ $\operatorname{det}(I+\mu \hat{\theta}(\xi))$. One verifies that

$$
\hat{d}(\mu, \xi)=[1+\mu \hat{\phi}(\xi)](1+\mu)-\mu \gamma^{2}(1-\xi)^{2-2 \alpha} / \hat{\phi}(\xi),
$$

with $\hat{\phi}(\xi)=(1-\xi)^{-\alpha} \hat{\psi}(\xi)$ as before. We may rearrange this as

$$
\hat{d}(\mu, \xi)=[1+\mu \hat{\phi}(\xi)][1+\mu \hat{e}(\mu, \xi)]
$$

with

$$
\hat{e}(\mu, \xi)=1-\gamma^{2}[1+\mu \hat{\phi}(\xi)]^{-1}(1-\xi)^{2-2 \alpha} / \hat{\phi}(\xi)
$$

Since

$$
(1-\xi)^{2-2 \alpha} / \hat{\phi}(\xi)=(1-\xi)^{2-\alpha} / \hat{\psi}(\xi) \in \Sigma(-\alpha-1)
$$

and

$$
[1+\mu \hat{\phi}(\xi)]^{-1}=1-\mu[1+\mu \hat{\phi}(\xi)]^{-1} \hat{\phi}(\xi)
$$

it follows that $\hat{e}(\mu, \xi)$ is an absolutely convergent power series for every $\mu \in S_{1}$. Also, using Lemma A.3, one verifies that $\mu \hat{e}(\mu, \xi) \in M\left(S_{1}, 1\right)$. Let $s=\sup \left\{\|e(\mu)\|_{l_{1}}\right.$ : $\left.\mu \in S_{1}\right\}$, and set $r=\min (1 / 2 s, 1)$. Then it follows from Lemma A.5 that 
$1-[1+\mu \hat{e}(\mu, \xi)]^{-1} \in M\left(S_{r}, 1\right)$ and that $[1+\mu \hat{e}(\mu, \xi)]^{-1} \hat{f}(\mu, \xi) \in M\left(S_{r, 1}\right)$ for every $f \in M\left(S_{r}, 1\right)$, and with Lemma A.6 that $\hat{f}(\mu, \xi) / \hat{d}(\mu, \xi) \in M\left(S_{r}, 1\right)$ for every $f \in M\left(S_{r}, 1\right)$. Finally, upon noting that

$$
\mu[1+\mu \hat{\phi}(\xi)]^{-1}(1-\xi)^{1-\alpha}=\mu[1+\mu \hat{\phi}(\xi)]^{-1} \hat{\phi}(\xi)(1-\xi) / \hat{\psi}(\xi)
$$

and

$$
\mu[1+\mu \hat{\phi}(\xi)]^{-1}(1-\xi)^{2-2 \alpha} / \hat{\phi}(\xi)=\mu[1+\mu \hat{\phi}(\xi)]^{-1} \hat{\phi}(\xi)(1-\xi)^{2} /[\hat{\psi}(\xi)]^{2},
$$

it follows from Lemma A.4 and Cramer's rule that $I-[I+\mu \hat{\theta}(\xi)]^{-1} \in M\left(S_{r}, 2\right)$. So

$$
\mu[I+\mu \hat{\theta}(\xi)]^{-1} \hat{\theta}(\xi)=I-[I+\mu \hat{\theta}(\xi)]^{-1} \in M\left(S_{r}, 2\right) .
$$

Proof of Lemma 5.3 for small $|\mu|$. We begin by recalling that $\hat{A}(\xi)=\hat{\Theta}(\xi) \hat{C}(\xi)$, with $C \in \Sigma(-\alpha-1)$, and that $\hat{A}(\xi)-\alpha(\alpha+1) \hat{\Theta}(\xi) \in \Sigma(-\alpha-1)$. Now consider $\hat{B}(\mu, \xi)=\mu[1+\mu \hat{A}(\xi)]^{-1} \hat{A}(\xi)$. Again, the familiar Kolodner manipulations give that

$$
\begin{aligned}
\hat{B}(\mu, \xi)=\{ & \left\{I+\mu\left[1+\mu_{\alpha} \hat{\Theta}(\xi)\right]^{-1}[\hat{A}(\xi)-\alpha(\alpha+1) \hat{\Theta}(\xi)]\right\}^{-1} \\
& \times \mu\left[I+\mu_{\alpha} \hat{\Theta}(\xi)\right]^{-1} \hat{\Theta}(\xi) \hat{C}(\xi),
\end{aligned}
$$

where $\mu_{\alpha}=\alpha(\alpha+1) \mu$. It follows from Lemma A.7 and the above that $\hat{E}(\mu, \xi)=$ $\left[1+\mu_{\alpha} \hat{\Theta}(\xi)\right]^{-1}[\hat{A}(\xi)-\alpha(\alpha+1) \Theta(\xi)]$ is absolutely convergent for all $\mu \in S_{r / \alpha(\alpha+1)}$, with $r$ as in Lemma A.7. Let $s=\sup \left\{\|E(\mu)\|_{l^{1}}: \mu \in S_{r / \alpha(\alpha+1)}\right\}$ and set $\rho=\min \{1 / 2 s, r / \alpha(\alpha+1)\}$. It now follows from Lemma A.7 that $\mu \hat{E}(\mu, \xi) \in$ $M\left(S_{\rho}, P+1\right)$ and from Lemma A.4 that $I-[I+\mu \hat{E}(\mu, \xi)]^{-1} \in M\left(S_{\rho}, P+1\right)$ and then finally that $\hat{B}(\mu, \xi) \in M\left(S_{\rho}, P+1\right)$.

Department of Mathematical Sciences

University of Delaware

Newark, Delaware 19716

1. C. T. H. BAKER \& G. F. MILlER (editors), Treatment of Integral Equations by Numerical Methods, Academic Press, London, 1982.

2. V. L. BAKKE \& Z. JACKIEWICZ, "Stability analysis of product $\theta$-methods for Abel integral equations of the second kind," Numer. Math., v. 48, 1986, pp. 127-136.

3. M. S. Berger, Nonlinearity and Functional Analysis, Academic Press, New York, 1977.

4. H. BRUNNER, "The numerical solution of weakly singular Volterra integral equations by collocation on graded meshes," Math. Comp., v. 45, 1985, pp. 417-437.

5. H. BRUNNER \& P. J. VAN DER HOUWEN, The Numerical Solution of Volterra Equations, North-Holland, Amsterdam, 1986.

6. N. G. DE BRUIJN \& P. ERDÖs, "On a recursion formula and some Tauberian theorems," J. Res. Nat. Bur. Standards, v. 50, 1953, pp. 161-164.

7. J. R. CANNON, The One-Dimensional Heat Equation, Addison-Wesley, Reading, Mass., 1984.

8. P. P. B. EgGERMONT, "A generalization of W. A. J. Luxemburg's asymptotic problem concerning the Laplace transform," Indag. Math., v. 42, 1981, pp. 257-265.

9. P. P. B. Eggermont, "On monotone Abel-Volterra integral equations on the half line," Numer. Math., v. 52, 1988, pp. 65-79.

10. P. P. B. Eggermont, "Galerkin methods for Abel-type integral equations of the first kind," SIAM J. Numer. Anal., v. 25, 1988, pp. 1093-1117.

11. P. P. B. EgGERMONT, "Approximation of non-smooth solutions of Abel-Volterra integral equations," manuscript, 1986.

12. A. ERDÉLYI et al., Higher Transcendental Functions, Vol. 3, McGraw-Hill, New York, 1955.

13. G. A. HUFFORD, "An integral equation approach to the problem of wave propagation over an irregular surface," J. Math. Mech., v. 9, 1952, pp. 391-404. 
14. D. Kershaw, "Some results for Abel-Volterra equations of the second kind," in [1], pp. 273-282 and 459-461.

15. I. I. KOLODNER, "Equations of Hammerstein in Hilbert space," J. Math. Mech., v. 13, 1964, pp. 701-750.

16. S.-O. LONDEN, "On some nonintegrable Volterra kernels with integrable resolvents including some applications to Riesz potentials," J. Integral Equations, v. 10, 1985, pp. 241-289.

17. CH. LUBICH, "Fractional linear multistep methods for Abel-Volterra integral equations of the second kind," Math. Comp., v. 45, 1985, pp. 463-469.

18. CH. LUBICH, "On the numerical solution of Volterra equations with unbounded nonlinearity," J. Integral Equations, v. 10, 1986, pp. 175-183.

19. W. A. J. LUXEMBURG, "An asymptotic problem concerning the Laplace transform," Applicable Anal., v. 9, 1979, pp. 61-70.

20. C. C. MEI \& E. O. TUCK, "Forward scattering by long thin bodies," SIAM J. Appl. Math., v. 39, 1980, pp. 178-191.

21. O. NEVANLINNA, "On the stability of discrete Volterra equations," in [1], pp. 139-147.

22. S. L. PAVERI-FONTANA \& R. RIGACCI, "A singularly perturbed singular integro-differential problem from analytical chemistry," in Numerical Analysis of Singular Perturbation Problems (P. W. Hemker and J. J. H. Miller, eds.), Academic Press, New York, 1979, pp. 475-484.

23. E. M. STEIN \& G. WEISS, Introduction to Fourier Analysis on Euclidean Spaces, Princeton Univ. Press, Princeton, N.J., 1971.

24. H. J. J. TE RIELE, "Collocation methods for weakly singular second kind Volterra integral equations with nonsmooth solutions," IMA J. Numer. Anal., v. 2, 1982, pp. 437-449. 